\title{
POTENT AXIOMS
}

BY

\author{
MATTHEW FOREMAN
}

\begin{abstract}
This paper suggests alternatives to the ordinary large cardinal axioms of set theory. These axioms can be viewed as generalizations of large cardinals and exhibit many of the same phenomena. They are shown to imply the G.C.H., every set of reals in $L(\mathbf{R})$ is Lebesgue measurable, and various results in combinatorics, algebra and model theory.
\end{abstract}

Potent axioms. A great number of mathematical conjectures have been shown to be independent of the ordinary axioms of Zermelo-Frankel set theory with the axiom of choice. They include propositions from virtually every area of mathematics, including analysis [W], algebra [Sh2], topology and combinatorics. Apparently the only way of settling these questions is to strengthen ZFC with natural axioms that answer these questions.

Two major axiom systems have been proposed; determinacy axioms and large cardinal axioms. They have been successful to a large extent but have not answered many crucial questions such as the continuum hypothesis. This paper proposes a line of axioms that can be viewed as generalizations of large cardinal axioms. It is shown that they settle the G.C.H. (positively) as well as several classical questions in set theory. It is shown that they form a strict hierarchy in consistency strength, and a hueristic argument is given to show that they may be stronger in consistency strength than ordinary large cardinal axioms. The author conjectures that they imply determinacy axioms.

Large cardinal axioms generally are equivalent to the existence of monomorphisms $j$ from $V$ (the mathematical universe) into a transitive class $M$. These monomorphisms are induced by measures taking values in $\{0,1\}$.

Paraphrasing [SRK] these monomorphisms, viewed as symmetries, are motivated by resemblance properties, generalizing properties of $\omega$ (e.g. compactness) and reflection properties. The main difficulty with these large cardinal axioms is that the resemblance, reflection and generalization occurs among cardinals much larger than sets usually occurring in ordinary mathematics. With remarkable and notable exceptions there is a paucity of consequences of these axioms "down low".

A solution to these problems comes from allowing the measures inducing the monomorphisms to take values in a complete Boolean algebra. This idea is not new (see [J-P]). What is new here is the sets we put measures on and the development of the consequences of these measures as axioms.

Received by the editors October 16, 1984 and, in revised form, April 10, 1985.

1980 Mathematics Subject Classification. Primary 03E55; Secondary 03E35, 04A30. 
The generic monomorphisms induced by these axioms can send any regular cardinal to any larger regular cardinal. Thus, we get strong resemblance properties between any regular cardinals.

In a typical example we have a generic elementary embedding $j: V \rightarrow M \subseteq V^{b}$ where $\mathscr{B}$ is a complete Boolean algebra. The three parameters determining the characteristics of $j$ are the closure of $M$, where $j$ moves ordinals and the nature of the forcing $\mathscr{B}$ (such as the saturation properties of $\mathscr{B}$ ). We will get strong results by manipulating these three parameters.

We now state the notational conventions to hold in this paper. If $X$ is a set, we use $\mathscr{P}(X)$ for the power set of $X$. We will use $\kappa$ and $\lambda$ to denote cardinals and $[\kappa]^{\lambda}$ and $[\kappa]^{<\lambda}$ to denote all subsets of $\kappa$ of power $\lambda$ and of power $<\lambda$ respectively. We will use the abbreviation o.t. $x$ for the order type of $x$. We will use $\kappa^{\kappa}, 2^{\kappa}$ and $\kappa^{+\gamma}$ to denote the powers of $[\kappa]^{<\kappa}, \mathscr{P}(\kappa)$ and the $\gamma$ th successor cardinal of $\kappa$. If $x \subseteq O R$ is a set, then $\sup x$ is the supremum of $x$.

We will be concerned with ideals throughout the paper. If $\mathscr{I} \subseteq \mathscr{P}(Z)$ is an ideal we will say that $\mathscr{I}$ is an ideal on $Z$. The degree of completeness of $\mathscr{I}, \operatorname{comp}(\mathscr{I})$, will be the least $\alpha$ such that there are $\left\langle x_{\beta}: \beta<\alpha\right\rangle \subseteq \mathscr{I}$ and $\bigcup_{\beta<\alpha} x_{\beta} \notin \mathscr{I}$. A set $X$ in

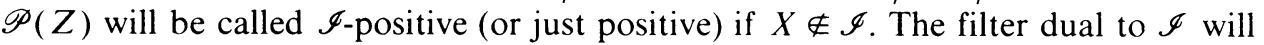
be denoted $\breve{\mathscr{I}}$. An ideal $\mathscr{I}$ will be called normal iff whenever $f: \mathscr{P}(Z) \rightarrow V$ and $S=\{x: f(x) \in x\}$ is positive then there is a $w$ and a positive set $T \subseteq S$ such that $T \subseteq\{x: f(x)=w\}$. If $Z=[\kappa]^{<\lambda}, \mathscr{I}$ is called fine iff, whenever $\alpha \in \kappa,\{x$ : $\alpha \in x\} \in \breve{\mathscr{I}}$. If $\mathscr{I}$ is an ideal on $[\kappa]^{\lambda}, \mathscr{I}$ is normal iff $\mathscr{I}$ is closed under diagonal union, e.g. if for all $\alpha \in \kappa, X_{\alpha} \in \mathscr{I}$ then $\nabla_{\alpha<\kappa} X_{\alpha}=\{x$ : there exists a $\beta \in x$, $\left.x \in X_{\beta}\right\} \in \mathscr{I}$. This is equivalent to $\breve{\mathscr{I}}$ being closed under diagonal intersection, i.e. if for all $\alpha \in \kappa, C_{\alpha} \in \check{\mathscr{I}}$ then $\Delta_{\alpha \in \kappa} C_{\alpha}=\left\{x\right.$ : for all $\left.\beta \in x, x \in C_{\beta}\right\} \in \check{\mathscr{I}}$. This is also equivalent to $\mathscr{I}$ being closed under double diagonal intersection, i.e. if $\left\{C_{\alpha \beta \beta}\right.$ : $\alpha, \beta \in \kappa\} \subseteq \breve{\mathscr{I}}$ then $\Delta_{\alpha, \beta<\kappa}=\left\{x\right.$ : for all $\left.\alpha, \beta \in x, x \in C_{\alpha, \beta}\right\} \in \mathscr{I}$. All ideals in this paper on $[\kappa]^{\lambda}$ will be normal, fine and countably complete.

We will often be concerned with more than one universe. To relativize a notion such as powerset to a universe $N$ we will use the superscript $N$, e.g. $\mathscr{P}(\kappa)^{N}$. We will use standard partial order and Boolean algebra notation interchangeably when forcing, with one exception that $p \Vdash q$ will mean that $p$ is a stronger forcing condition than $q$. If $\phi$ is a property then $p \| \phi$ means $p$ decides $\phi,\|\phi\|_{\mathbf{P}}$ will be the Boolean value of $\phi$ taken in $\mathscr{B}(\mathbf{P})$, the complete Boolean algebra generated by $\mathbf{P}$. We will drop the subscript $\mathbf{P}$ when it is obvious. We will use $\mathbf{P} \sim Q$ to mean that $\mathscr{B}(\mathbf{P})$ is isomorphic to $\mathscr{B}(Q)$. When forcing with $\mathbf{P}, G$ will be the generic generic object, i.e. a canonical term for the canonical generic object for $\mathbf{P}$. If $i: \mathbf{P} \rightarrow Q, i$ is called a neat embedding iff $i$ can be extended to a complete monomorphism $i: \mathscr{B}(\mathbf{P}) \rightarrow \mathscr{B}(Q)$. If $\pi: Q \rightarrow \mathbf{P}$ is an order preserving map such that $\pi(i(p))=p$ and $q \Vdash i(\pi(q))$ and for all $p \in \mathbf{P}$ and all $p^{\prime} \Vdash p$ there is a $q \in Q$ with $q \Vdash i(p)$ such that $\pi(q) \Vdash p^{\prime}$, then $i$ is a neat embedding and $\pi$ is called a projection. If $i: \mathbf{P} \hookrightarrow Q$ is a neat embedding and $G_{\mathbf{P}} \subseteq \mathbf{P}$ is generic, then we can form $\mathscr{B}(Q) / G_{\mathbf{P}}=\{b \in \mathscr{B}(Q)$ : for all $\left.p \in G_{\mathbf{P}}, i(p) \nvdash \neg b\right\}$ in the standard way. We will abuse notation and refer 
to this as $Q / \mathbf{P}$. If $q \in Q$ then $Q / q$ will be the part of $Q$ consisting of all conditions extending $q$. Similarly $Q /\langle\mathbf{P}, q\rangle$ are all conditions in $\mathscr{B}(Q) / G_{\mathbf{P}}$ extending $q$.

A particular partial ordering we will be interested in is $\operatorname{Col}(\kappa, \lambda)$, the Levy collapse of $\lambda$ to $\kappa^{+}$. We will use the notation $\operatorname{Col}(\kappa, \lambda]$ to mean the Levy partial ordering the makes $\lambda$ have cardinality $\kappa$. If $p \in \mathbf{P}_{\alpha}, \mathbf{P}_{\alpha}$ an iteration of partial orderings, then supp $p$ will be the support of $p$.

If $\mathscr{I}$ is an ideal on $Z$, we can form the Boolean algebra $\mathscr{P}(Z) / \mathscr{I}$. Forcing with the Boolean algebra gives an ultrafilter $G$ on $\mathscr{P}(Z)^{V}$. We can take the ultraproduct of $V$ using functions $f: Z \rightarrow V$ such that $f \in V$. Let $M$ denote the ultraproduct. Elements of $M$ are equivalence classes $[f]_{G}$ of functions $f \in V, f: Z \rightarrow V$. Los' theorem applies in this case and hence there is a canonical embedding $j: V \rightarrow M$. We will call $j$ the embedding induced by $\mathscr{I}$. If $M$ is well founded then we replace $M$ by its unique transitive isomorph. If $M$ is the ultraproduct over any field of sets $\mathscr{F}$ and $f$ is a function defined on a set in $\mathscr{F}$ then $[f]_{M}$ is the set in $M$ that $f$ represents. If $f: Z \rightarrow V$ then $[f]_{\mathscr{I}}=\{g$ : for some set $C \in \check{\mathscr{I}}, f=g$ on $C\}$. $\mathscr{I}$ will be called precipitous iff, wherever $G \subseteq \mathscr{P}(Z) / \mathscr{I}$ is generic, $M$ is well-founded.

In certain sections of this paper we will be concerned with model theory. All of our models will be in a countable language. If $\mathfrak{A}=\left\langle A ; U, f_{i}\right\rangle_{i \in \omega}$ is a structure with a distinguished predicate $U$, then the type of $\mathfrak{A}$ is $(\kappa, \lambda)$ iff $|A|=\kappa$ and $|U|=\lambda$. If $\mathfrak{A}, \mathfrak{B}$ are structures in the same language, we will write $\mathfrak{A} \prec \mathfrak{B}$ iff $\mathfrak{A}$ is an elementary substructure of $\mathfrak{B}$. If $H$ is a $\operatorname{graph}$ then $\operatorname{chrom}(H)$, the chromatic number of $H$, is the least number of colors it takes to color $H$ so that no two adjacent nodes have the same color.

If $\mathscr{F}$ is a family of functions defined on a cardinal $\kappa$, then $\mathscr{F}$ is called almost disjoint iff for all $f, g \in \mathscr{F}$ there is a $\gamma<\kappa$ such that for all $\alpha, \gamma<\alpha<\kappa$, $f(\alpha) \neq g(\alpha)$. If $j: V \rightarrow M$ is an elementary embedding then $j$ is called generic iff there is a partial ordering $\mathbf{P}$ such that $j$ is definable in $V^{\mathbf{P}}$.

1. In this section $M$ will be a transitive model of ZFC unless otherwise stated. We now give our first axiom.

Weak Axiom of Resemblance. For all finite sequences of uncountable regular cardinals $\lambda_{1}<\cdots<\lambda_{n}$ there is a generic elementary embedding $j: V \rightarrow M$ such that $j\left(\boldsymbol{\aleph}_{i}\right)=\lambda_{i}$ for $1 \leqslant i \leqslant n$.

The motivation for the weak resemblance axiom is that regular cardinals should in some vague sense be indiscernible. In $\S 4$ we will show the following theorem:

THEOREM 1. If the G.C.H. holds and for all $n, \boldsymbol{\aleph}_{n}$ carries a normal, $\boldsymbol{\aleph}_{n}$-complete $\aleph_{n+1}$-saturated ideal, then the weak axiom of resemblance holds.

This theorem coupled with the following theorem of the author (see [F1]) shows that the weak resemblance property is consistent relative to large cardinals.

THEOREM. If ZFC + there is a huge cardinal is consistent then so is ZFC+ G.C.H. + every regular cardinal $\kappa$ carries a normal, $\kappa$-complete, $\kappa^{+}{ }^{-}$-saturated ideal. 
We now turn to a stronger, more useful axiom:

Axiom of Resemblance. For all finite sequences of uncountable regular cardinals $\lambda_{1}<\cdots<\lambda_{n}$ there is a generic elementary $j: V \rightarrow M$ such that $j\left(\boldsymbol{\aleph}_{i}\right)=\lambda_{i}$ for $1 \leqslant i \leqslant n$ and $\mathscr{P}\left(\lambda_{n}\right)^{V} \subseteq M$.

We shall show that local versions of the Axiom of Resemblance are consistent, but the axiom as stated is not known to be consistent from any assumptions. We prove the next easy theorem to show the power of this axiom.

Theorem 2 (ZFC + Axiom of Resemblance). If $2^{\boldsymbol{\kappa}_{0}} \leqslant \boldsymbol{\aleph}_{n}$ then, for all cardinals $\kappa, 2^{\kappa} \leqslant \kappa^{+n}$.

Proof. We show that if $2^{\boldsymbol{\kappa}_{0}} \leqslant \boldsymbol{\kappa}_{n}$ then, for all regular $\kappa, \kappa^{\kappa} \leqslant \kappa^{+n-1}$. Let $\kappa$ be a cardinal such that $\kappa^{\kappa}>\kappa^{+n-1}$. Let $X=\left\langle x_{\alpha}: \alpha\left\langle\kappa^{+n}\right\rangle\right.$ be a wellordering of a sequence of elements of $[\kappa]^{<\kappa}$ of order type $\kappa^{+n}$. By the Axiom of Resemblance there is an elementary embedding $j: V \rightarrow M$ such that $j\left(\boldsymbol{\aleph}_{1}\right)=\kappa$ and $j\left(\boldsymbol{\aleph}_{i+1}\right)=\kappa^{+i}$ for $i \leqslant n$ and $\mathscr{P}\left(\kappa^{+n}\right)^{V} \subseteq M$. (Hence $X \in M$.) Since $j\left(\boldsymbol{\aleph}_{i+1}\right)=\kappa^{+i}, M \vDash \kappa^{+i}=$ $\boldsymbol{\aleph}_{i+1}$ and thus $M \vDash X$ is a sequence of elements of $\boldsymbol{\aleph}_{i}^{\aleph_{i}}$ of length $\boldsymbol{\aleph}_{n+1}$. But by the elementarity of $j, M \vDash \boldsymbol{\aleph}_{i}^{\boldsymbol{\aleph}_{1}} \leqslant \boldsymbol{\aleph}_{n}$, a contradiction.

Later we shall we how to state the resemblance axiom in terms of ideals.

Definition. Let $j: V \rightarrow M$ be a generic elementary embedding. Let $\kappa_{0}=\operatorname{crit}(j)$ and $\kappa_{i+1}=j\left(\kappa_{i}\right)$. Then $j$ is said to be generically $n$-huge iff $j^{\prime \prime} \kappa_{n} \in M$. We call $j$ generically almost $n$-huge iff, for each $\alpha<\kappa_{n}, j^{\prime \prime} \alpha \in M$.

Lemma 3. If $j: V \rightarrow M$ is any elementary embedding such that $j^{\prime \prime} \lambda \in M$, then $\mathscr{P}(\lambda)^{V} \subseteq M$.

Proof. Let $Y \subseteq \lambda$. In $M$, consider $\pi: j^{\prime \prime} \lambda \rightarrow \lambda$ the unique order preserving collapsing map. Then $Y=\pi^{\prime \prime}\left(j(Y) \cap j^{\prime \prime} \lambda\right)$.

We now have discussed two of the three parameters used in the definition of the Potent Axioms. We turn to the third, the nature of the forcing.

If $j: V \rightarrow M$ is a generic elementary embedding gotten by forcing with $\mathbf{P}$ and $j^{\prime \prime} \lambda \in M$ then in $V$ we get a normal and fine ideal on $[\lambda]^{\leqslant \lambda}$ given by the following: if $X \subseteq[\lambda]^{\leqslant \lambda}, X \in \mathscr{I}$ iff $\left\|j^{\prime \prime} \lambda \in I(X)\right\|_{\mathbf{P}}=0$. If $\kappa=\operatorname{crit}(j)$ then these ideals are $\kappa$-complete.

Definition. An ideal $\mathscr{I}$ on $Z$ will be called $\mu$-saturated iff $\mathscr{P}(Z) / \mathscr{I}$ satisfies the $\mu$ chain condition. The ideal $\mathscr{I}$ will be called $\mu$-centered iff there are filters $\left\langle\mathscr{F}_{\alpha}\right.$ : $\alpha<\mu\rangle$ on $\mathscr{P}(Z) / \mathscr{I}$ such that for each positive set $X \subseteq Z$ there is an $\alpha$ such that $X \in \mathscr{F}_{\alpha}$. Finally, $\mathscr{I}$ will be called $\mu$-dense iff $\mathscr{P}(Z) / \mathscr{I}$ has a dense set of cardinality $\mu$.

Lemma 4. Suppose that $\mathscr{I}$ is an ideal in a Boolean algebra $\mathscr{C}$ and $i: \mathscr{C} \rightarrow \mathscr{B}$ is a homomorphism such that $\mathscr{I}=\{c: i(c)=0\}$. Then (i) if $\mathscr{B}$ is $\kappa$-saturated, so is $\mathscr{C} / \mathscr{I}$, (ii) if $\mathscr{B}$ is $\kappa$-centered then so is $\mathscr{C} / \mathscr{I}$.

Proof. Note that $i$ induces a monomorphism from $\mathscr{C} / \mathscr{I}$ into $\mathscr{B}$. The lemma follows from the definitions. 
The importance of Lemma 4 is that it shows in many cases that the relevant properties of a partial ordering $\mathbf{P}$ are inherited by the ideal $\mathscr{I} \subseteq \mathscr{P}\left([\lambda]^{\lambda}\right)$.

If $\pi: Z \rightarrow Z^{\prime}$ is a surjection and $J$ is a $\kappa$-complete, $\lambda$-saturated ideal on $Z$ let $i$ : $\mathscr{P}\left(Z^{\prime}\right) \rightarrow \mathscr{P}(Z) / J$ be defined by $i\left(X^{\prime}\right)=\left[\left\{x \in Z: \pi(x) \in X^{\prime}\right\}\right]_{J}$. Then by Lemma $4, i$ induces an ideal on $Z^{\prime}$ that is $\kappa$-complete and $\lambda$-saturated. Further, if $X \subseteq Z \in \breve{J}$ then $\pi^{\prime \prime} X \in \breve{\mathscr{I}}$. We will occasionally call $\mathscr{I}$ by $\pi(J)$.

Definition. Suppose $\mathscr{I}$ is an ideal on $[\lambda]^{\kappa}$ and $\lambda^{\prime} \leqslant \lambda, \kappa^{\prime}<\kappa$. $\mathscr{I}$ is said to concentrate on $\left[\lambda^{\prime}\right]^{\kappa^{\prime}}$ if $\left\{x\right.$ : o.t. $\left.x \cap \lambda^{\prime}=\kappa^{\prime}\right\} \in \breve{\mathscr{I}} . \mathscr{I}$ is said to concentrate on $\left[\lambda^{\prime}\right]^{\left|\kappa^{\prime}\right|}$ iff $\left\{x:\left|x \cap \lambda^{\prime}\right|=\kappa^{\prime}\right\} \in \breve{\mathscr{I}}$. If $\mathscr{I}$ concentrates on $\left[\lambda^{\prime}\right]^{\kappa^{\prime}}$ then we can define a map $\pi$ : $[\lambda]^{\kappa} \rightarrow\left[\lambda^{\prime}\right]^{\kappa^{\prime}}$ defined on a set in $\breve{\mathscr{I}}$ by $\pi(x)=x \cap \lambda^{\prime}$. Then $\pi(\mathscr{I})$ is a nontrivial ideal on $\left[\lambda^{\prime}\right]^{\kappa^{\prime}}$ and if $\mathscr{I}$ is normal and fine then $\pi(\mathscr{I})$ is normal and fine. Finally, if $\mathscr{I}$ is an ideal on $[\lambda]^{\leqslant \lambda}$ concentrating on $[\lambda]^{\kappa}$ we view it as an ideal on $[\lambda]^{\kappa}$ since $[\lambda]^{\kappa}$ is a set of measure one.

It may not necessarily be true in Lemma 4 that if $\mathscr{B}$ is $\mu$-dense then $\mathscr{C} / \mathscr{I}$ is $\mu$-dense. However, in the context we are interested in, it is true.

Let $J$ be a $\mu$-dense, $\mu$ complete, normal and fine ideal on $[\lambda]^{\kappa}$. If $J$ concentrates on $\left[\lambda^{\prime}\right]^{\kappa^{\prime}}$, let $\mathscr{I}$ be the projection of $J$ onto an ideal on $\left[\lambda^{\prime}\right]^{\kappa^{\prime}}$. We claim that $\mathscr{I}$ is $\mu$-dense.

Let $D \subseteq \mathscr{P}\left([\lambda]^{\kappa}\right) / J$ be a dense set of cardinality $\mu$. For each $d \in D$, choose a representative $A_{d} \subseteq[\lambda]^{\kappa}$. Let $\pi:[\lambda]^{\kappa} \rightarrow\left[\lambda^{\prime}\right]^{\kappa^{\prime}}$ be the projection map.

Fix a $d \in D$. For each set $C \in \breve{J}$, let $B_{c}=\sim \pi\left(C \cap A_{d}\right)$. By the fineness and $\mu^{+}$-saturation of $\mathscr{I}$ there is a sequence $\left\langle b_{\alpha}^{d}: \alpha<\mu\right\rangle \subseteq\left\langle B_{c}: c \in J\right\rangle$ such that every $B_{c} \subseteq \nabla_{\alpha<\mu} b_{\alpha}^{d}$ modulo $\mathscr{I}$. For each $b_{\alpha}^{d}$ there is a $C_{\alpha}^{d}$ such that $b_{\alpha}^{d}=\sim \pi\left(C_{\alpha}^{d} \cap A_{d}\right)$. Then $\pi\left(\Delta_{\alpha<\mu} C_{\alpha}^{d} \cap A_{d}\right) \cap \nabla_{\alpha<\mu} b_{\alpha}^{d} \in \mathscr{I}$. Further, $\Delta_{\alpha<\mu} C_{\alpha} \cap A_{d} \notin J$ and $\pi\left(\Delta_{\alpha<\mu} C_{\alpha}^{d} \cap A_{d}\right) \cup \nabla_{\alpha<\mu} b_{\alpha}^{d} \in \check{\mathscr{I}}$.

If $T \in \mathscr{P}\left(\left[\kappa^{\prime}\right]^{\lambda^{\prime}}\right)$ and $i(T) \geqslant d$ in $\mathscr{P}\left([\kappa]^{\lambda}\right) / J$ then there is a $C$ such that $i(T) \supseteq A_{d} \cap C$. Hence $T \supseteq \pi\left(A_{d} \cap C\right)$. But $B_{c}=\sim \pi\left(A_{d} \cap C\right)$ is disjoint modulo $\mathscr{I}$ from $\pi\left(\Delta_{\alpha<\mu} C_{\alpha}^{d} \cap A_{d}\right)$. Hence $T \supseteq \pi\left(\Delta_{\alpha<\mu} C_{\alpha}^{d} \cap A_{d}\right)$ modulo $\mathscr{I}$.

Thus if we let $E=\left\{[e]_{\mathscr{g}}\right.$ : for some $\left.d \in D, e=\pi\left(\Delta_{\alpha<\mu} C_{\alpha}^{d} \cap A_{d}\right)\right\}$ then $E$ is a dense set in $\mathscr{P}\left(\left[\lambda^{\prime}\right]^{\kappa}\right) / \mathscr{I}$ of cardinality $\mu$.

Similarly we can show that $J$ projects to a $\mu$-dense ideal on $\mu$.

Lemma 5. Suppose $\mathscr{I}$ is a normal, fine, precipitous ideal on $[\lambda]^{\kappa}$ concentrating on $\left[\lambda^{\prime}\right]^{\kappa^{\prime}}$. Let $j: V \rightarrow M$ be the embedding induced by $\mathscr{I}$. Then

(a) $j^{\prime \prime} \lambda \in M$,

(b) $j(\kappa)=\lambda$ and $j\left(\kappa^{\prime}\right)=\lambda^{\prime}$,

(c) there is a positive set $X \subseteq[\lambda]^{\kappa}$ such that if $X \in G, \operatorname{crit}(j)=\operatorname{comp}(\mathscr{I})$, and

(d) if $G$ is the generic object for $\mathscr{P}\left([\lambda]^{\kappa}\right) / \mathscr{I}$ then $X \in G$ iff $j^{\prime \prime} \lambda \in j(X)$.

Proof. (a) Let id: $[\lambda]^{\kappa} \rightarrow[\lambda]^{\kappa}$. Using normality and fineness it is easy to see that $[\mathrm{id}]_{M}=j^{\prime \prime} \lambda$.

(b) Since $\left\{x \in[\lambda]^{\kappa}\right.$ : o.t. $\left.x=\kappa\right\} \in \check{\mathscr{I}}$, Los' theorem implies that o.t. $j^{\prime \prime} \lambda=j(\kappa)$. Further, $\left\{x \in[\lambda]^{\kappa}\right.$ : o.t. $\left.x \cap \lambda^{\prime}=\kappa^{\prime}\right\} \in \breve{\mathscr{I}}$, so o.t. $\left(j^{\prime \prime} \lambda \cap j\left(\lambda^{\prime}\right)\right)=j\left(\kappa^{\prime}\right)$. But $j^{\prime \prime} \lambda \cap$ $j\left(\lambda^{\prime}\right)=j^{\prime \prime} \lambda^{\prime}$. Hence $j\left(\kappa^{\prime}\right)=$ o.t. $j^{\prime \prime} \lambda^{\prime}=\lambda^{\prime}$. 
(c) Standard arguments show that $\operatorname{crit}(j) \geqslant \operatorname{comp}(\mathscr{I})$. Let $\left\langle Y_{\alpha}: \alpha<\gamma\right\rangle(\gamma=$ $\operatorname{comp}(\mathscr{I}))$ be an increasing sequence such that $Y_{\alpha} \in \mathscr{I}$ but $X=\bigcup Y_{\alpha} \notin \mathscr{I}$. Let $f$ : $X \rightarrow \gamma$ be defined by $f(x)=$ least $\alpha, x \in Y_{\alpha}$. Then it is easy to check that if $X \in G$ then $[f]>j(\alpha)$ for each $\alpha<\gamma$ and $[f]<j(\gamma)$.

(d) Suppose $X \in G$. Then $\{x$ : $\operatorname{id}(x) \in X\} \in G$. Hence by (a), $j^{\prime \prime} \lambda \in j(X)$. Conversely, if $\{x: \operatorname{id}(x) \notin X\} \in G$ then $j^{\prime \prime} \lambda \in j(\tilde{X})$.

REMARKS. (a) We can prove similarly that if $\mathscr{I}$ concentrates on $[\lambda]^{<\kappa}$ then $j(\kappa)>\lambda$.

(b) One can easily see by similar arguments the converse to Lemma 5(b) i.e. if $[X] \in \mathscr{P}\left([\lambda]^{\kappa}\right) / \mathscr{I}$ and $[X] \vDash j\left(\kappa^{\prime}\right)=\lambda^{\prime}$ then there is a set $C \in \breve{\mathscr{I}}$ such that, for all $x \in C \cap X$, o.t. $x \cap \lambda^{\prime}=\kappa^{\prime}$.

(c) If $\mathscr{I}$ concentrates on $\left[\lambda^{\prime}\right]^{\left|\kappa^{\prime}\right|}$ instead of $\left[\lambda^{\prime}\right]^{\kappa^{\prime}}$ then one sees that $M \vDash\left|\lambda^{\prime}\right|=j\left(\kappa^{\prime}\right)$.

COROllary 6. Let $\lambda_{0}<\lambda_{1}<\cdots<\lambda_{n}$ be a sequence of regular cardinals. Let $\mathscr{I}$ be a $\lambda_{0}$-complete, normal, fine, precipitous ideal on $\left[\lambda_{n}\right]^{\lambda_{n-1}}$ concentrating on $\left[\lambda_{k}\right]^{\lambda_{k-1}}$ for $1 \leqslant k \leqslant n$. If $j: V \rightarrow M$ is the generic elementary embedding induced by $\mathscr{I}$ then $j$ is generically $n$-huge.

Proof. Note that $\kappa_{i}=\lambda_{i}$.

COROLlary 7. We can formulate resemblance properties in terms of ideals. Suppose for all regular cardinals $\kappa_{1}<\cdots<\kappa_{n}$ there is a precipitous ideal on $\left[\kappa_{n}\right]^{\kappa_{n}}$ concentrating on $\left[\kappa_{i}\right]^{\kappa_{i}}$. Then the Axiom of Resemblance holds.

Proof. This follows immediately from Lemmas 5 and 3.

We now turn to ideals that give $M$ stronger closure properties.

Definition. Let $\mathscr{I} \subseteq \mathscr{P}(Z)$ be an ideal. Then $\mathscr{I}$ has the disjointing property iff whenever $A \subseteq \mathscr{P}(Z) / \mathscr{I}$ is a maximal antichain, there is a pairwise disjoint partition of $Z,\left\langle X_{a}: a \in A\right\rangle$ such that $\left[X_{a}\right]_{\mathscr{g}}=a$.

The following proposition is standard (see [J1]).

Proposition 8. Suppose $\mathscr{I} \subseteq \mathscr{P}(Z)$ is a $\kappa$-complete, $\kappa^{+}$-saturated ideal on $Z$, then $\mathscr{I}$ has the disjointing property.

The author would like to thank M. Magidor for pointing out the following proposition which simplified several proofs in this paper.

Proposition 9. Suppose $\mathscr{I} \subseteq \mathscr{P}\left([\lambda]^{\kappa}\right)$ is a normal, fine $\lambda^{+}$-saturated ideal. Then $\mathscr{I}$ has the disjointing property.

Note. This is also true for $[\lambda]^{<\kappa}$.

Proof. Let $A \subseteq \mathscr{P}\left([\lambda]^{\kappa}\right) / \mathscr{I}$ be a maximal antichain. By the saturation of $\mathscr{I}$ we can assume that $A=\left\{\left[a_{\alpha}\right]: \alpha<\lambda\right\}$, where $a_{\alpha} \subseteq[\lambda]^{\kappa}$ are arbitrarily chosen representatives of the elements of $A$. By fineness, we can assume that if $x \in a_{\alpha}$ then $\alpha \in x$. If $\alpha, \beta \in \lambda$, then there is a set $C_{\alpha, \beta} \in \breve{\mathscr{I}}$ such that $C_{\alpha, \beta} \cap a_{\alpha} \cap a_{\beta}=\varnothing$. Let $C=\Delta_{\alpha, \beta<\lambda} C_{\alpha, \beta}$. We claim that $C \cap a_{\alpha} \cap a_{\beta}=\varnothing$. Otherwise, there is an $x$ such that $x \in a_{\alpha} \cap a_{\beta}$, and so $\alpha, \beta \in x$. But then $x \in C_{\alpha, \beta}$, a contradiction. Letting $a_{\alpha}^{\prime}=a_{\alpha} \cap C$, then $\left[a_{\alpha}^{\prime}\right]=\left[a_{\alpha}\right]$ and the $a_{\alpha}^{\prime}$ are disjoint. 
The following lemma is a reworking of standard lemmas.

Lemma 10. Suppose $\mathscr{I}$ is a countably complete ideal on $Z, \mathbf{P}=\mathscr{P}(Z) / \mathscr{I}$ and $\mathscr{I}$ has the disjointing property. Then:

(i) If $\tau$ is a term in $V^{\mathbf{P}}$ for an element of $M$ then there is a function $f: Z \rightarrow V$, $f \in V$ such that $\left\|\tau=[f]_{M}\right\|=1$.

(ii) $\mathscr{I}$ is precipitous.

(iii) If $Z=[\lambda]^{\kappa}$ and $\mathscr{I}$ is normal and fine, then $M^{\lambda} \cap V[G] \subseteq M$.

Proof. (i) Let $\tau$ be a term for an element of $M$. Then there is a maximal antichain $A$ such that for all $a \in A$ there is a function $f_{a}: Z \rightarrow V, f_{a} \in V$ and $a \Vdash\left[f_{a}\right]_{M}=\tau$. By the disjointing property we can assume that the elements of $A$ are pairwise disjoint. Glue together the $f_{a}$ 's by $f(x)=f_{a}(x)$ where $a$ is the unique element of $A$ such that $x \in a$. Then $\left\|[f]_{M}=\tau\right\|=1$.

(ii) Suppose there is a descending chain of $M$-ordinals $\left\langle\tau_{n}: n \in \omega\right\rangle \subseteq M$. By (i), there is a sequence of functions $\left\langle f_{n}: n \in \omega\right\rangle \in V$ such that $\left\|\left[f_{n}\right]_{M}=\tau_{n}\right\|=1$. Hence there is a $p \in \mathbf{P}$ such that for all $n$ there is a set $C_{n} \in \breve{\mathscr{I}}$ such that, for all $x \in C_{n} \cap P, f_{n+1}(x) \in f_{n}(x)$. Let $C=\cap C_{n}$, then $C \in \check{\mathscr{I}}$ and, for any $x \in C$, $\left\langle f_{n+1}(x): n \in \omega\right\rangle$ forms a descending sequence of ordinals in $V$, a contradiction.

(iii) Let $\left\langle\tau_{\alpha}: \alpha \in \lambda\right\rangle$ be a term for a $\lambda$-sequence in $V[G]$ of elements of $M$.

By (i) we can find in $V$ a sequence $\left\langle f_{\alpha}: \alpha<\lambda\right\rangle$ such that $\left\|\left[f_{\alpha}\right]_{M}=\tau_{\alpha}\right\|=1$. Let $g$ : $[\lambda]^{\kappa} \rightarrow V$ be defined by $g(x)=\left\{f_{\alpha}(x): \alpha \in x\right\}$. It is easy to verify using normality and fineness that $[g]_{M}=\left\{\tau_{\alpha}^{V[G]}: \alpha<\lambda\right\}$.

COROllary 11. Suppose $\mathscr{I}$ is a normal, fine, precipitous ideal on $[\lambda]^{\leqslant \lambda}$ such that $\mathscr{I}$ has the disjointing property. Then for each $\alpha<\lambda^{+}, j^{\prime \prime} \alpha \in M$.

The saturation of an ideal gives a great deal of information about where the cardinals go under the generic embeddings.

Proposition 12. Suppose $\kappa$ is a successor cardinal and $\mathscr{I}$ is a $\kappa$-complete $\kappa^{+}-$ saturated ideal on $[\lambda]^{\gamma}$ such that $\operatorname{comp}(I)=\kappa$. Then for each $n \in \omega$ such that $\kappa^{+n} \leqslant \lambda, \mathscr{I}$ concentrates on $\left[\kappa^{+n}\right]^{\kappa^{+n-1}}$. Hence by Corollary 11 the embedding $j$ induced by $\mathscr{I}$ is a generic almost- $(n+1)$-huge embedding.

Proof. Since $\mathscr{I}$ is $\kappa^{+}$-saturated, forcing with $\mathbf{P}=\mathscr{P}\left([\lambda]^{\gamma}\right) / \mathscr{I}$ preserves the cardinals $\kappa^{+n}$ for each $n$. We now trace where the cardinals are moved by $j: V \rightarrow M$, the embedding induced by the ideal $\mathscr{I}$. Since $\operatorname{comp}(I)=\kappa, \operatorname{crit}(j)=\kappa$. Note that $P\left(\kappa^{+n}\right)^{V} \subseteq M$, so if $\kappa=\delta^{+}$, then $\left(\kappa^{+n}\right)^{V} \leqslant\left(\delta^{+n+1}\right)^{M}$ and if $\eta<\kappa^{+n}$ is not a cardinal in $V$, it is not a cardinal in $M$. Further, if $\nu<j(\kappa), M \vDash|\nu|=\delta$. Hence, by saturation, $j(\kappa) \leqslant\left(\kappa^{+}\right)^{V}$. Since $M \vDash j(\kappa)$ is a cardinal we get $j(\kappa)=\kappa^{+}$. Similar computations give $j\left(\kappa^{+i}\right)=\kappa^{+i+1}$ for each $i$ such that $\kappa^{+i+1} \leqslant \lambda$. Hence, for each $n, \kappa^{+n} \leqslant \lambda, \kappa_{n}=\kappa^{+n}$ and $j$ is at least a generic almost $(n+1)$-huge embedding.

REMARK 13. If we know in advance that $\mathscr{I}$ concentrates on $\left[\kappa^{+n}\right]^{\kappa^{+n-1}}$ and $\operatorname{comp}(\mathscr{I})=\kappa$, we could drop the saturation hypothesis and show $j\left(\kappa^{+i}\right)=\kappa^{i+1}$ for $i<n$. Hence $\mathscr{I}$ concentrates on $\left[\kappa^{+i+1}\right]^{\kappa+i}$. 
To see this we count cardinals. By Lemma 5, $j\left(\kappa^{+n-1}\right)=\left(\kappa^{+n}\right)^{V}$. Hence $\left(\kappa^{+n}\right)^{V}$ $=\left(\delta^{+n}\right)^{M}$. Thus there must be $n-1$ cardinals in $M$ between $\delta$ and $\left(\kappa^{+n}\right)^{V}$. Since $\mathscr{P}\left(\kappa^{+n}\right)^{V} \subseteq M$ and $\kappa$ is moved by $j$, there is nowhere for $\kappa^{+i}$ to go via $j$ but to $\left(\kappa^{+i+1}\right)^{V}$. Thus any $\mathscr{I}$ concentrating on $\left[\kappa^{+n}\right]^{\kappa^{* n}}$ yields a generic $n$-huge embedding.

It is also clear that if $\lambda$ is a cardinal and $\mathscr{I}$ concentrates on $[\lambda]^{\leqslant \lambda}$ and $\mathscr{I}$ is $\lambda$-saturated then, for some cardinal $\kappa \leqslant \lambda, \mathscr{I}$ concentrates on $[\lambda]^{\kappa}$. (Otherwise, by Lemma $5, \lambda$ would not be a cardinal in $M$.)

We now turn our attention to a technical device we shall use in a couple of places in this paper. Iterated ultrapowers were used by Gaifman [G] and developed extensively by Kunen [K1]. They were used in a generic context by Solovay and later by Wagon.

We will give a cursory development to prove a couple of main lemmas.

Let $\mathscr{I}$ be an ideal on a set $Z$. Define by induction ideals $\mathscr{I}^{n}$ on $Z^{n}$ (the cartesian product of $n$-copies of $Z$ ).

If $X \subseteq Z \times Z^{n}$ then $X \in \mathscr{I}^{n+1}$ iff $\left\{x:\left\{\vec{y} \in Z^{n}: x^{n} \vec{y} \in X\right\} \in \mathscr{I}^{n}\right\} \in \check{\mathscr{I}}$. If $\theta$ is an ordinal, let $\mathscr{P}_{f}\left(Z^{\theta}\right)$ be the collection of subsets of $Z^{\theta}$ with finite support, i.e. $\left\{X \subseteq Z^{\theta}\right.$ : there are $\alpha_{1} \cdots \alpha_{n}<\theta$ such that for $f, g \in Z^{\theta}$ if $\left(f\left(\alpha_{1}\right) \cdots f\left(\alpha_{n}\right)\right)=$ $\left(g\left(\alpha_{1}\right) \cdots g\left(\alpha_{n}\right)\right)$ then $f \in X$ iff $\left.g \in X\right\}$. In the definition above, $\alpha_{1} \cdots \alpha_{n}$ will be called a support for $X$. If $X$ has support $\alpha_{1} \cdots \alpha_{n}$ then $X$ can be identified with $\left\{f \uparrow\left(\alpha_{1} \cdots \alpha_{n}\right): f \in X\right\}$ and hence canonically with a subset of $Z^{n}$. We define an ideal $\mathscr{I}^{\theta}$ on $\mathscr{P}_{f}\left(Z^{\theta}\right)$ by saying that $X \in \mathscr{I}^{\theta}$ iff, when we canonically identify $X$ with a subset of $Z^{n}, X \in \mathscr{I}^{n}$. We note that this definition does not depend on the choice of a support for $X$.

Having defined $\mathscr{I}^{\theta}$ on $\mathscr{P}_{f}\left(Z^{\theta}\right)$, we note that $\mathscr{I}^{\theta}$ is an ideal and we can form the partial ordering $\mathbf{P}_{\theta}=\mathscr{P}_{f}\left(Z^{\theta}\right) / \mathscr{I}^{\theta}$. We similarly defined $F n_{f}\left(Z^{\theta}\right)$ to be the class of functions $F: \kappa^{\theta} \rightarrow V$ such that there are $\alpha_{1} \cdots \alpha_{n} \in \theta$ such that if $f, g \in \kappa^{\theta}$ and $f \uparrow\left(\alpha_{1} \cdots \alpha_{n}\right)=g \uparrow\left(\alpha_{1} \cdots \alpha_{n}\right)$ then $F(f)=F(g)$.

If we force with $\mathbf{P}_{\theta}$ then we get an ultrafilter $G_{\theta} \subseteq \mathbf{P}_{\theta}$ and can form the ultrapower of $V$ by functions in $F_{f}(Z) \cap V$. The result is a model $M_{\theta}$ which we will also call $\mathrm{Ult}_{\theta}(V, \mathscr{I})$. Los' theorem can be proven in this case and we get an elementary embedding $i_{0, \theta}: V \rightarrow M_{\theta}$. (So far, $M_{\theta}$ is not necessarily well founded.) If $\alpha<\beta$ and $G_{\beta} \subseteq \mathscr{P}_{f}\left(Z^{\beta}\right)$ is generic then $G_{\beta}$ induces an ultrafilter $G_{\alpha}$ on $\mathscr{P}_{f}\left(Z^{\alpha}\right)$. We can check that $G_{\alpha}$ is generic for $\mathscr{P}_{f}\left(Z^{\alpha}\right) / \mathscr{I}^{\alpha}$. Further we get a natural mapping $j^{*}$ from $F n_{f}\left(Z^{\alpha}\right)$ into $F n_{f}\left(Z^{\beta}\right)$ such that $j^{*}$ induces an elementary embedding $i_{\alpha, \beta}: M_{\alpha} \rightarrow$ $M_{\beta}$. Note that if $\beta$ is a limit then $M_{\beta}$ is the direct limit of $\left\langle M_{\alpha}: \alpha<\beta\right\rangle$ by the maps $\left\langle i_{\alpha, \alpha^{\prime}}: \alpha<\alpha^{\prime}<\beta\right\rangle$.

Solovay pointed out that an argument by Gaifman suitably modified gave the following lemma:

Lemma 14. If $\mathscr{I}$ is a precipitous ideal on $Z$ and $G_{\alpha} \subseteq \mathscr{P}_{f}\left(Z^{\alpha}\right) / \mathscr{I}^{\alpha}$ is generic, then $M_{\alpha}$ is well founded.

Proof. We first remark that a variation on Kunen's arguments show that if $\beta<\alpha, \alpha=\beta+\gamma, M_{\beta}$ is well founded and $G_{\alpha}$ is $V$-generic then there are $G_{\beta}$ and $G^{\gamma}$ 
such that

(1) $G_{\beta} \subseteq \mathscr{P}_{f}\left(Z^{\beta}\right) / \mathscr{I}^{\beta}$ is generic,

(2) $G^{\gamma} \subseteq\left\{\mathscr{P}_{f}\left(i_{0, \beta}(Z)^{\gamma}\right) / i_{0, \beta}(\mathscr{I})^{\gamma}\right\}^{M_{\beta}}$ is $M_{\beta}$-generic,

(3) $\operatorname{Ult}_{\gamma}\left(M_{\beta}, G^{\gamma}\right) \simeq \operatorname{Ult}_{\beta+\gamma}\left(V, G_{\alpha}\right)$ (where $\operatorname{Ult}_{\gamma}\left(M_{\beta}, G^{\gamma}\right)$ is the ultrapower taken inside $M_{\beta}$ ).

(The main point here is the $M_{\beta}$-genericity of $G^{\gamma}$. However, a dense set $D \subseteq$ $\mathscr{P}_{f}\left(i_{0, \beta}(Z)^{\gamma}\right) / i_{0, \beta}(\mathscr{I})^{\gamma}$ such that $D \in M$ can be pulled back to a dense set $D^{\prime} \subseteq$ $\mathscr{P}_{f}\left(Z^{\beta+\gamma}\right) / \mathscr{I}^{\beta+\gamma}$.)

Let $\alpha$ be the least possible ordinal such that $M_{\alpha}$ is not well founded and let $\xi$ be the least possible ordinal such that $i_{0, \alpha}(\xi)$ is above a descending $\omega$-sequence of ordinals in $M_{\alpha}$. Let $p \in \mathscr{P}_{f}\left(Z^{\alpha}\right) / \mathscr{I}^{\alpha}$ be a condition that forces that $M_{\alpha}$ is not well founded and $\xi$ is the least ordinal above a descending $\omega$-sequence of ordinals in $M_{\alpha}$.

We note that $\alpha$ cannot be a successor since if $\alpha=\beta+1, M_{\beta}$ is well founded and $M_{\beta} \vDash i_{0, \beta}(\mathscr{I})$ is precipitous. Hence $M_{\beta+1}=\operatorname{Ult}\left(M_{\beta}, i_{0, \beta}(\mathscr{I})\right)$ is well founded.

Thus $\alpha$ is a limit ordinal. Since the support of $p$ is finite there is some stage $\beta<\alpha, \alpha=\beta+\gamma$ such that

$$
\begin{aligned}
& M_{\beta} \vDash \| \gamma \text { is the least place such that } \mathrm{Ult}_{\gamma}\left(M_{\beta}, G^{\gamma}\right) \\
& \text { is not well founded and } i_{0, \beta}(\xi) \text { is the least ordinal } \\
& \delta \text { such that } i_{\beta, \beta+\gamma}(\delta) \text { is above a descending } \\
& \omega \text {-sequence in } \mathrm{Ult}_{\gamma}\left(M_{\beta}, G^{\gamma}\right) \|=1 .
\end{aligned}
$$

(The Boolean value is taken in $M_{\beta}$ in the partial ordering $\mathscr{P}_{f}\left(i_{0, \beta}(Z)^{\gamma}\right) / i_{0, \beta}(\mathscr{I})^{\gamma}$.)

Now, since $i_{0, \alpha}(\xi)$ is above a descending sequence $\xi_{1}>\xi_{2}>\cdots$ and $M_{\alpha}=$ $\lim _{\rightarrow}\left\langle M_{\beta}: \beta<\alpha\right\rangle$ there is a $\beta<\alpha, \alpha=\beta+\gamma$ such that $\xi_{1}=i_{\beta, \alpha}\left(\xi^{\prime}\right)$ for some $\xi^{\prime} \in M_{\beta}, \xi^{\prime}<i_{0, \beta}(\xi)$. Hence there is a condition $q$ in $\left\{\mathscr{P}_{f}\left(i_{0, \beta}(Z)^{\gamma}\right) / i_{0, \beta}(\mathscr{I})^{\gamma}\right\}^{M_{\beta}}$ such that $M_{\beta} \vDash q \Vdash i_{\beta, \beta+\gamma}\left(\xi^{\prime}\right)$ is above a descending $\omega$-sequence of ordinals in $\operatorname{Ult}\left(M_{\beta}, G^{\gamma}\right)$. This however contradicts (*).

2. The hierarchy theorem. All of our ideals from now on will be normal, fine and countably complete.

We now show that our axioms form a strict hierarchy in consistency strength.

THEOREM 15 (ZFC). Suppose that $\kappa$ is a successor of a regular cardinal, $\kappa \geqslant \boldsymbol{\aleph}_{2}$ and there is a $\kappa$-complete, $\kappa^{+}$-saturated (respectively $\kappa$-centered or $\kappa$-dense) ideal on $\left[\kappa^{+n+1}\right]^{\kappa^{+n}}(\leqslant n \leqslant \omega)$. Then there is a transitive set model $N$ of ZFC such that $\kappa^{+n} \subseteq N$ and $N \vDash$ there is a $\kappa$-complete, $\kappa^{+}$-saturated (respectively $\kappa$-centered or $\kappa$-dense) ideal on $\left[\kappa^{+n}\right]^{+n-1}$. Further we can make $N$ have the same cardinals as $V$ does up to $\kappa^{+n}$.

RemarKs. (a) The same is true for $\kappa=\boldsymbol{\aleph}_{1}$ if $2^{\boldsymbol{\kappa}_{n}} \leqslant \boldsymbol{\aleph}_{n+1}$. We just eliminates the first step of the proof.

(b) If $n=0$, then the proof shows that there is a transitive set model of ZFC + there is a $\kappa^{+}$-saturated (or $\kappa$-centered) ideal on $\kappa$. This is true even for $\kappa=\boldsymbol{\aleph}_{1}$. 
(c) This theorem is of the form " $\mathrm{ZFC}+$ there is a generic almost- $n$-huge embedding implies the consistency of $\mathrm{ZFC}+$ there is an almost- $(n-1)$-huge embedding".

We use a theorem of Shelah [Sh].

TheOREM. Suppose $\kappa=\delta^{+}$and $\mathscr{I}$ is a $\kappa$-complete $\kappa^{+}$-saturated ideal on $\kappa$. Then $\{\alpha<\kappa: \operatorname{cof}(\alpha)=\operatorname{cof}(\delta)\} \in \check{\mathscr{I}}$.

Proof of TheOrem 15. The author is grateful to M. Magidor for simplifying some aspects of this proof.

Let $\mathbf{P}=\mathscr{P}\left(\left[\kappa^{+n+1}\right]^{\kappa^{+n}}\right) / \mathscr{I}$ where $\mathscr{I}$ is $\kappa^{+}$-saturated. Let $j: V \rightarrow M$ be the embedding induced by $\mathscr{I}$.

Let $\pi:\left[\kappa^{+n+1}\right]^{\kappa^{* n}} \rightarrow \kappa$ be the projection. ( $\pi$ is defined on a set in $\breve{\mathscr{I}}$.) Then by Lemma 4 the ideal $\pi(\mathscr{I})$ on $\kappa$ is $\kappa^{+}$-saturated. Hence by Shelah's theorem. $\pi(\mathscr{I})$ concentrates on ordinals of cofinality $>\omega$. Hence, $\{x: x \cap \kappa$ has cofinality $>\omega\} \in \breve{\mathscr{I}}$. Hence, in $M, \operatorname{cof}(\kappa)>\omega$.

We claim that $j^{\prime \prime} \kappa^{+n+1}$ is $\omega$-closed. (This is where we use $\kappa \geqslant \omega_{2}$.) Otherwise there is a sequence $\left\langle j\left(\alpha_{n}\right): n \in \omega\right\rangle$ such that $\sup \left\langle j\left(\alpha_{n}\right): n \in \omega\right\rangle \notin j^{\prime \prime} \kappa^{+n+1}$. Let $\alpha=\sup _{n \in \omega} \alpha_{n}$. Then the cofinality of $\alpha$ in $V$ is not $\omega$ but cofinality of $\alpha$ in $V^{\mathbf{P}}$ is $\omega$.

Let $\xi=\operatorname{cof}(\alpha)^{V}$. We cannot have $\xi>\kappa$ since $\mathbf{P}$ is $\kappa^{+}$-saturated. Since ${ }^{\xi} M \cap V[G]$ $\subseteq M, \omega=\operatorname{cof}(\xi)^{V[G]}=\operatorname{cof}(\xi)^{M}$. Hence by Shelah's theorem, $\xi \neq \kappa$. If $\xi<\kappa$, then $j(\xi)=\xi$ hence by the elementarity of $j, \operatorname{cof}(\xi)^{V}=\omega$ but this is a contradiction. Hence $j^{\prime \prime} \kappa^{+n+1}$ is $\omega$-closed.

We use the $\omega$-closure of $j^{\prime \prime} \kappa^{+n+1}$ to get a set $X$ in $\breve{\mathscr{I}}$ such that sup: $\left\{x \cap \kappa^{+n}\right.$ : $x \in X\} \rightarrow \kappa^{+n}$ is 1-1. If $\pi^{*}:\left[\kappa^{+n+1}\right]^{\kappa^{+n}} \rightarrow\left[\kappa^{+n}\right]^{\kappa^{+n-1}}$ is defined by $\pi^{*}(x)=x \cap$ $\kappa^{+n}$, this implies that $\pi^{*}(\mathscr{I})$ has a set of measure one of cardinality $\kappa^{+n}$.

Let $\left\langle A_{\alpha}: \alpha<\kappa^{+n}\right\rangle$ be a partition of $\left\{\alpha<\kappa^{+n}: \operatorname{cof}(\alpha)=\omega\right\}$ into $\kappa^{+n}$ disjoint stationary sets.

Claim. For a set $X \in \check{\mathscr{I}}, x \in X$ iff $x \cap \kappa^{+n}=\left\{\alpha: A_{\alpha}\right.$ is stationary in Sup $\left.x\right\}$.

To see the claim: In $M$ we examine $j^{\prime \prime} \kappa^{+n}$. Let $\gamma=\sup j^{\prime \prime} \kappa^{+n}$. Then, by the saturation, $\operatorname{cof}(\gamma)>\omega$. It is enough to see that for each $\beta<j\left(\kappa^{+n}\right)$, if $B$ is the $\beta$ th set in the sequence $j\left(\left\langle A_{\alpha}: \alpha<\kappa^{+n}\right\rangle\right)$ then $B \cap \gamma$ is stationary iff $\beta=j(\alpha)$ for some $\alpha$.

Suppose $\beta=j(\alpha)$. Then $B=j\left(A_{\alpha}\right)$. Let $C \subseteq \gamma$ be a closed unbounded set. Since $j^{\prime \prime} \kappa^{+n+1}$ is $\omega$-closed, $C \cap j^{\prime \prime} \kappa^{+n}$ is $\omega$-closed and unbounded in $\gamma$. Hence $C^{\prime}=j^{-1}(C)$ is $\omega$-closed and unbounded in $\kappa^{+n}$. Since $\mathbf{P}$ is $\kappa^{+}$-c.c. there is a $D \subseteq C^{\prime}, D \omega$-closed and unbounded in $\kappa^{+n}$ and $D \in V$. But then $D \cap A_{\alpha} \neq \varnothing$, hence $C \cap j\left(A_{\alpha}\right) \neq \varnothing$ and $j\left(A_{\alpha}\right)$ is stationary as desired.

Now suppose that $B \cap \gamma$ is stationary in $\gamma$. Then, since $j^{\prime \prime} \kappa^{+n}$ is $\omega$-closed there is a $\delta \in j^{\prime \prime} \kappa^{+n} \cap B$, say $\delta=j\left(\delta^{\prime}\right)$. Choose the unique $\alpha$ such that $\delta^{\prime} \in A_{\alpha}$. Then $j\left(A_{\alpha}\right) \cap B \neq \varnothing$. Hence, since $j\left(\left\langle A_{\alpha}: \alpha<\kappa^{+n}\right\rangle\right)$ is a pairwise disjoint sequence, $j\left(A_{\alpha}\right)=B$, so $j(\alpha)=\beta$. This proves the claim.

By Proposition 12, $J=\pi^{*}(\mathscr{I})$ is a nontrivial ideal on $\left[\kappa^{+n}\right]^{\kappa^{+n \cdot 1}}$. By the claim, there is a set $X \in \breve{J}$ such that sup: $X \rightarrow \kappa^{+n}$ is 1-1. 
Let $M_{\omega}$ be the $\omega$ th iterate of $\mathscr{I}$. Let $\delta=j_{0, \omega}(\kappa)$. For each $\alpha<\kappa^{+n}$, let $f_{\alpha}$ be a bijection between $\alpha$ and $|\alpha|$. Let $F: \kappa^{+n} \times \kappa^{+n} \rightarrow \kappa^{+n}$ be given by $F(\alpha, \beta)=f_{\alpha}(\beta)$. Let $N=L_{\delta}[X, F, J]$. (If $\mathscr{I}$ is $\kappa$-centered we let $N=L_{\delta}[X, F, J, C]$ where $C$ is a $\kappa$-centering of $\mathscr{P}\left(\left[\kappa^{+n}\right]^{\kappa^{+n-1}}\right) / J$. If $\mathscr{I}$ is $\kappa$-dense we let $N=L_{\delta}[X, F, J, D]$ where $D$ is a dense set in $\mathscr{P}\left(\left[\kappa^{+n}\right]^{\kappa^{+n-1}}\right) / J$ of cardinality $\kappa$.) We will show that $N$ is a model of ZFC $+J$ is a $\kappa^{+}$-saturated (respectively $\kappa$-centered or $\kappa$-dense) ideal on $\left[\kappa^{+n}\right]^{\kappa^{+n-1}}$.

(We are being slightly careless here: we are coding $X$ by the set $\tilde{X}=\{\langle\alpha, \beta\rangle$ : $\beta \in x$ for the unique $x \in X$ such that $\sup x=\alpha\}$ and $N$ is really $L_{\delta}[\tilde{X}, F, J]$. We construct from $\tilde{X}$ to make sure that $X \in N$, otherwise there will probably not be a set of measure one for $J$. We need to have a well-ordered set of measure one in order to have a choice hold in $N$.)

It is clear that since $F \in N, N$ has the same cardinals below $\kappa^{+n}$ as $V$ does.

Claim. $\left|\mathscr{P}\left(\kappa^{+n}\right)^{N}\right| \leqslant \kappa^{+n+1}$.

Proof. We imitate the proof that if $U$ is a normal ultrafilter on $\lambda$ then $L[U] \Vdash 2^{\lambda}=\lambda^{+}$(see $\left.[\mathbf{S}]\right)$. Consider $L[X, F, J]$. Since $L[X, F, J] \supseteq N$ it is enough to see that $\left|\mathscr{P}\left(\kappa^{+n}\right)^{L[X, F, J]}\right| \leqslant \kappa^{+n+1}$.

Let $x \subseteq \kappa^{+n}, x \in L[X, F, J]$. We show that $L[X, F, J] \vDash \mid\left\{y \subseteq \kappa^{+n}: y\right.$ is constructed before $x\} \mid \leqslant \kappa^{+n}$.

Working inside $L[X, F, J]$, choose $\alpha$ such that $x \in L_{\alpha}[X, F, J]$. Let $M \prec$ $L_{\alpha}[X, F, J]$ be such that $X \cup \kappa^{+n} \cup\left\{\kappa^{+n}\right\} \cup\{x\} \subseteq M$ and $|M|=\kappa^{+n}$. Let $M^{\prime}$ be the transitive collapse of $M$. It is easy to check that $M^{\prime}=L_{\gamma}\left[X, F, J^{\prime}\right]$ for some $J^{\prime}$ and also that $J^{\prime}=J$. Hence $M^{\prime} \supseteq\left\{y \subseteq \kappa^{+n}: y \in L[X, F, J]\right.$ and $y$ is constructed before $x\}$. But $\left|M^{\prime}\right|=\kappa^{+n}$, hence $\mid\left\{y \in L[X, F, J]: y \subseteq \kappa^{+n}\right.$ and $y$ is constructed before $x\} \mid \leqslant \kappa^{+n}$. This proves the claim.

We will now show that $N \vDash Z$ ZFC.

Since $\left|\mathscr{P}\left(\kappa^{+n}\right)\right|^{N} \leqslant \kappa^{+n+1}, J \cap N, X$ and $F$ are in each model $M_{i}$ for $i<\omega$. Consider $M_{i}$ for $i \geqslant n+1$. Then $j_{0, i}(\kappa)>\kappa^{+n+1}$, by $i$ consecutive applications of Lemma 5. Since $N \in M_{i}$ and $j_{0, i}(\kappa)$ is a regular cardinal in $M_{i}$, it follows that $j_{0, i}(\kappa)$ is a regular cardinal in $N$. Thus $N$ has a cofinal sequence of regular cardinals. Since $N \vDash V=L[A]$ for a bounded set $A$, it will be enough to show that there is no set $\mathbf{a} \in N$ and no formula $\phi(x, y)$ with parameters from $N$ such that

$N \vDash \phi$ defines a functional from a cofinally into the ordinals.

Let $A$ be a subset of $\kappa^{+n+1}$ canonically coding $X, F$ and $J \cap N$. Then $A \in M_{\omega}$, since $A \in M_{i}$ for each $i$ and for large enough $i, j_{i, i+1}(A)=A$. Hence $N \in M_{\omega}$. If $\phi^{N}: a \rightarrow \delta$ and $\phi$ is cofinal then $\phi$ is definable in $M_{\omega}$. Hence $M_{\omega} \vDash \delta$ is singular. This, however, is a contradiction, since $\delta=j_{0, \omega}(\kappa)$ and $\kappa$ is regular in $V$. Hence $M_{\omega} \vDash \kappa$ is regular. This completes the proof of Theorem 15.

It is not known to the author how to get a consistency hierarchy relating generic-huge cardinals to generic almost huge cardinals. A typical conjecture along these lines might be the following

Question. Suppose there is an $\boldsymbol{\aleph}_{2}$-complete, precipitous ideal on $\left[\boldsymbol{\aleph}_{3}\right]^{\boldsymbol{\aleph}_{2}}$. Is there an $\boldsymbol{\aleph}_{2}$-complete, $\boldsymbol{\aleph}_{3}$-saturated ideal on $\boldsymbol{\aleph}_{2}$ ?

3. We now turn our attention to impossibility results and known results showing the consistency of these ideals relative to large cardinal assumptions. 
A major attraction of these axioms is that the known impossibility results for the ideals are significantly weaker than the impossibility results for their large cardinal analogues.

The following proposition is crucial in all of the impossibility arguments. A stronger theorem was known earlier to Magidor.

Proposition 16. Suppose $\mathscr{I}$ is a precipitous ideal on $[\lambda]^{\kappa}$ concentrating on $\left[\lambda^{\prime}\right]^{\left|\kappa^{\prime}\right|}$. Then every structure $\mathfrak{A}$ of type $\left(\lambda, \lambda^{\prime}\right)$ has a proper elementary substructure of type $\left(\kappa, \kappa^{\prime}\right)$.

Proof. Note that this makes sense even if $\lambda=\lambda^{\prime}=\kappa=\kappa^{\prime}$. Consider $j: V \rightarrow M$ induced by $\mathscr{I}$. Let $\mathfrak{A}$ be a structure of type $\left(\lambda, \lambda^{\prime}\right)$, say $\mathfrak{U}=\left\langle\lambda ; \lambda^{\prime}, f_{i}\right\rangle_{i \in \omega}$. We can assume that $\mathfrak{A}$ is fully skolemized. By Lemma $5, j^{\prime \prime} \lambda \in M$ and $j^{\prime \prime} \lambda$ has order type $j(\kappa)$. Hence, in $M j^{\prime \prime} \mathfrak{A}$ is a proper elementary substructure of $j(\mathfrak{A})$ of type $\left(j(\kappa), j\left(\kappa^{\prime}\right)\right)$. Thus, by elementarity, $\mathfrak{A}$ has an elementary substructure of type $\left(\kappa, \kappa^{\prime}\right)$.

Thus it is possible to have many versions of Chang's Conjecture and Jonsson Cardinals by assuming the appropriate ideal. This is an example of an application of second order ideals that does not follow from ordinary ideals on cardinals. In [F-M1] it is shown, relative to an almost-huge cardinal, that it is consistent to have $\square_{\aleph_{n}}$ and and $\boldsymbol{\aleph}_{n+1}$-saturated ideal on $\boldsymbol{\aleph}_{n}$. It is known that $\square_{\boldsymbol{\aleph}_{1}}$ implies that Chang's Conjecture fails at $\left(\boldsymbol{\aleph}_{2}, \boldsymbol{\aleph}_{1}\right)$. Hence saturated ideals on $\kappa$ do not yield Chang's Conjecture.

Woodin has shown that if $\kappa$ is the successor of a regular cardinal then there is a Jonsson algebra on $\kappa$, i.e. a structure of cardinality $\kappa$ with no proper elementary substructures of cardinality $\kappa$. By Proposition 16 , we know that this implies that there are no precipitous ideals on $[\lambda]^{\lambda}$ where $\lambda$ is the successor of a regular cardinal. Magidor has improved this result. Let $\mathscr{I}$ be an ideal on $[\lambda]^{\gamma}$. Let $j: V \rightarrow M$ be the induced elementary embedding. Let $\left\langle\kappa_{i}: i \in \omega\right\rangle$ be defined in the usual way.

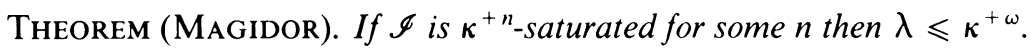

A proof of this appears in the appendix of this paper.

COROllaRY. If $\mathscr{I}$ is an $\boldsymbol{\aleph}_{n}$-saturated ideal on $[\lambda]^{\gamma}$ then $\lambda \leqslant \boldsymbol{\aleph}_{\omega}$.

The author would like to point out that this still leaves open the possibility of a precipitous ideal on $\left[\boldsymbol{\kappa}_{\omega+1}\right]^{\boldsymbol{\kappa}_{\omega+1}}$ with critical point some $\boldsymbol{\aleph}_{n}$. The closure of $M$ in such a model would be two power-set operations higher than allowed by conventional large cardinals (i.e. if $j: V \rightarrow M$ is a nongeneric elementary embedding then $\mathscr{P}\left(\sup \left\langle\kappa_{i}: i \in \omega\right\rangle\right) \nsubseteq M$. However if there is a precipitous ideal on $\left[\boldsymbol{\kappa}_{\omega+1}\right]^{\boldsymbol{\kappa}_{\omega+1}}$ then $\boldsymbol{\aleph}_{\omega}=\sup \left\langle\kappa_{i}: i \in \omega\right\rangle$ and $\left.\mathscr{P}\left(\boldsymbol{\aleph}_{\omega+1}\right) \subseteq M\right)$. Shelah has shown that if $\boldsymbol{\aleph}_{\omega+1}$ is Jonsson, then $2^{\boldsymbol{N}_{0}} \geqslant \boldsymbol{\aleph}_{\omega+1}$. Hence, by the remarks above, if there is a precipitous ideal on $\left[\boldsymbol{\kappa}_{\omega+1}\right]^{\boldsymbol{\kappa}_{\omega+1}}$ the C.H. fails badly.

The following lemma is well known (see [K2]).

Lemma 17. Suppose $\mathbf{P}$ is a $\lambda$-saturated partial ordering and in $V^{\mathbf{P}}$ we have an ultrafilter $U$ on $\mathscr{P}(Z)^{V}$. Then in $V$, if we let $\mathscr{I}=\left\{x:\|x \in U\|_{\mathbf{P}}=0\right\}, \mathscr{I}$ is $\lambda$-saturated. 
We now discuss some positive results on the existence of certain ideals.

If $j: V \rightarrow M$ is an elementary embedding and $Z \in V$ then for any $y \in j(Z)$ we can define the ultrafilter on $Z$ induced by $j$ and $y$ by

$$
U=\{X \subset Z ; y \in j(X)\} .
$$

Let $j$ be an embedding definable in $V$ and suppose that $U$ is induced by $j$ and $y$ and $U$ is nonprincipal. Suppose that $\mathbf{P} \in V$ is partial ordering such that there is a $q \in j(\mathbf{P})$ :

$q \Vdash$ there are generic ultrafilters $\hat{G} \subseteq j(\mathbf{P})$ and $G \subseteq \mathbf{P}$ such that $q \in \hat{G}$ and, for all $p \in \mathbf{P}, p \in G$ iff $j(p) \in \hat{G}$.

Then if $\hat{G}$ and $G$ are such ultrafilters, $j$ can be extended to $\hat{j}: V[G] \rightarrow M[\hat{G}]$. Further, in $V$ we can find a projection map from $\mathscr{B}(j(\mathbf{P})) / q \rightarrow \mathscr{B}(\mathbf{P})$. In $V[G]$ we get an ideal $\mathscr{I}$ on $Z$ by $X \in \mathscr{I}$ iff $\|y \in \hat{j}(X)\|_{j(\mathbf{P}) /\langle\mathbf{P}, q\rangle}=0$. We note that the new ideal extends $\breve{U}$. Further we get a commutative triangle

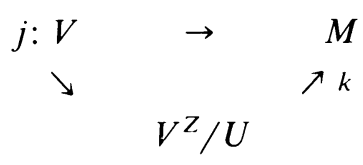

where $k\left([f]_{U}\right)=j(f)(y)$.

The following theorem is well known in the context of ideals on a cardinal. The proof is the same so we omit it.

Theorem. Let $j, U, \mathbf{P}, q, y$ and $\mathscr{I}$ be as above. Suppose that $j(\mathbf{P}) \subseteq$ range $k$. Then

(a) $\mathscr{I}$ is precipitous,

(b) if $Z=[\lambda]^{\kappa}$ and $U$ is normal and fine, then $\mathscr{I}$ is normal and fine.

We now give several examples of precipitous and saturated ideals on second order objects. We will use the following well-known lemma (see [K2]).

LEMMA 18. Suppose $\mathbf{P}$ is a $2^{|Z|}$-c.c. partial ordering and $Q$ is a $<2^{|Z|}$-closed partial ordering in $V^{\mathbf{P}}$ and in $V^{\mathbf{P} * Q}$ there is a $\kappa$-complete, normal and fine ultrafilter on $\mathscr{P}(Z)^{V}$. Then in $V^{\mathbf{P}}$ there is a $\mathrm{k}$-complete normal and fine ultrafilter on $\mathscr{P}(Z)^{V}$.

Proof. Build a tower of conditions in $Q$ deciding, for each subset $X$ of $Z$, whether $X$ is in or out of the ultrafilter.

EXAMPLE 1. Suppose that $\kappa$ is a supercompact cardinal. Let $\mathbf{P}$ be a partial ordering. Suppose that whenever $\lambda>\kappa, p \in \mathbf{P}$, there is a normal, fine, ultrafilter $U_{\lambda}$ on $Z=[\lambda]^{<\kappa}$ such that if $j: V \rightarrow V^{Z} / U_{\lambda}=M_{\lambda}$ is the elementary embedding induced by $U_{\lambda}$ there is a $q \in j(\mathbf{P})$

$$
\begin{aligned}
& q \Vdash_{j(\mathbf{P})} \text { there is a } G \subseteq \mathbf{P} \text { and a } \hat{G} \subseteq j(\mathbf{P}), \\
& q \in \hat{G}, p \in G \text { and, for all } r \in \mathbf{P}, r \in G \text { iff } j(r) \in \hat{G} .
\end{aligned}
$$

Then, in $V[G]$ we know that for all $\lambda \geqslant \kappa$ there is a normal, fine precipitous ideal on $[\lambda]^{<\kappa}$.

In particular, if $\mathbf{P}=\operatorname{Col}(\omega, \kappa)$ then $V^{\mathbf{P}} \vDash$ for all cardinals $\lambda$ there is a precipitous ideal on $[\lambda]^{<\omega_{1}}$. 
These ideals were considered by Magidor in [M1].

EXAMPLE 2. In [K2], Kunen started in a model $V$ with a measurable cardinal and constructed a partial ordering $\mathbf{P}$ such that in $V^{\mathbf{P}}$ there is a normal, fine, $\kappa$-complete, $\kappa$-saturated ideal on an inaccessible cardinal $\kappa$. (P adds a Cohen-generic subset to each inaccessible $\alpha<\kappa$, and then adds a homogeneous Suslin tree to $\kappa$.)

In $V^{\mathrm{P}}$ there is a Suslin tree $T$ on $\kappa$ such that, after forcing with $T, \kappa$ becomes measurable.

If $\kappa$ were supercompact in $V$ and, for example, we add subsets to $\alpha<\kappa$ according to a Laver function, finishing by adding a homogeneous Suslin tree $T$ to $\kappa$ then, after forcing with $T$ in $V^{\mathbf{P}}, \kappa$ would become supercompact. Thus in $V^{\mathbf{P}}$, for each $\lambda>\kappa$ there would be a normal, fine, $\kappa$-saturated $\kappa$-complete ideal on $[\lambda]^{<\kappa}$.

ExAmple 3. This model was also constructed by Kunen in [K2]. Let $\kappa_{0}$ be a huge cardinal, $U$ a normal, fine and $\kappa_{0}$-complete ultrafilter on $\left[\kappa_{1}\right]^{\kappa_{0}}$, and $j: V \rightarrow$ $V^{\left[\kappa_{1}\right]^{\kappa_{0}}} / U \approx M$ be the embedding induced by $U$. Kunen built a partial ordering $\mathbf{P}$ such that in $V^{\mathbf{P}}, \kappa_{0}=\boldsymbol{\aleph}_{1}, \kappa_{1}=\boldsymbol{\aleph}_{2}$ and there is a $q \in j(\mathbf{P})(q$ is the mastercondition $)$,

$$
\begin{aligned}
& q \Vdash \text { there is a } G \subseteq \mathbf{P} \text {, and a } \hat{G} \subseteq j(\mathbf{P}), q \in \hat{G}, \\
& \text { and, for all } p \in \mathbf{P}, p \in G \text { iff } j(p) \in \hat{G} \text {. }
\end{aligned}
$$

Hence $j$ can be extended to $\hat{j}: V[G] \rightarrow M[\hat{G}]$. Since $M$ is given by the ultraproduct by $U$, which is the ultrafilter induced by $j$ and $j^{\prime \prime} \kappa_{1}$, it is easy to check that $V^{\mathbf{P}}$ satisfies the criterion for having a precipitous ideal $\mathscr{I}^{*}$ on $Z=\left(\left[\kappa_{1}\right]^{\kappa_{0}}\right)^{V}$. We can now get a precipitous ideal on $\left(\left[\kappa_{1}\right]^{\kappa_{0}}\right)^{V^{\mathrm{P}}}$ by letting $\mathscr{I}: \mathscr{I}^{*} \cup\{\tilde{Z}\}$. Since $\kappa_{0}=\boldsymbol{\aleph}_{1}$ and $\kappa_{1}=\boldsymbol{\kappa}_{2}$ we get a normal, fine precipitous ideal on $\left[\boldsymbol{\kappa}_{2}\right]^{\boldsymbol{\kappa}_{1}}$.

Laver modified Kunen's model to get an $\boldsymbol{\aleph}_{n+1}$-saturated ideal on $\boldsymbol{\aleph}_{n}$. In Laver's model $\kappa_{0}$ becomes $\boldsymbol{\aleph}_{n}$ and $\kappa_{1}$ becomes $\boldsymbol{\kappa}_{n+1}$. It is easy to check that in this model there is a normal, fine, $\boldsymbol{\aleph}_{n}$-complete precipitous ideal on $\left[\boldsymbol{\kappa}_{n+1}\right]^{\boldsymbol{\aleph}_{n}}$.

EXAmple 4. These techniques can be modified to produce many of the Resemblance Axiom type ideals. For example, it is easy to modify Kunen's model so that $\kappa_{0}$ becomes $\aleph_{1}$ and $\kappa_{1}$ becomes $\aleph_{n}$. In this case if our original embedding is slightly more than a huge embedding we get a precipitous ideal on $\left[\boldsymbol{\kappa}_{2 n-1}\right]^{\aleph_{n}}$ concentrating on $\left[\boldsymbol{\aleph}_{n+i-1}\right]^{\boldsymbol{\aleph}_{i}}$ for each $1 \leqslant i \leqslant n$.

We can get many Resemblance Axiom type ideals in this manner.

EXAMPLE 5. We consider a model that was constructed by the author in [F2]. Let $j: V \rightarrow M$ be a two-huge embedding induced by the ultraproduct of $\left[\kappa_{2}\right]^{\kappa_{1}}$ by a normal, fine $\kappa_{0}$-complete ultrafilter concentrating on $\left[\kappa_{1}\right]^{\kappa_{0}}$. For each $n \in \omega$, the author constructed a partial ordering $\mathbf{P}$ such that, in $V^{\mathbf{P}}, \kappa_{0}=\boldsymbol{\aleph}_{n}, \kappa_{1}=\boldsymbol{\aleph}_{n+1}$, $\kappa_{2}=\boldsymbol{\aleph}_{n+2}$. It is eash to verify that the induced ideal satisfies the criterion for precipitousness. Hence, we get the consistency of a normal, fine, precipitous $\aleph_{n}$-complete ideal on $\left[\boldsymbol{\kappa}_{n+2}\right]^{\boldsymbol{\kappa}_{n+1}}$ concentrating on $\left[\boldsymbol{\kappa}_{n+1}\right]^{\boldsymbol{\aleph}_{n}}$. Thus we have the consistency of a generic two-huge embedding.

EXAMPLE 6. We do not have many examples of saturated second order ideals at this time. To explore the only example we know of we must examine the model in [F2] more closely. 
We start with a two-huge embedding $j: V \rightarrow M$ with $j\left(\kappa_{0}\right)=\kappa_{1}, j\left(\kappa_{1}\right)=\kappa_{2}$, $\kappa_{0}=\operatorname{crit}(j)$. In [F2] a partial ordering $\mathbf{P} * \mathbf{R} * S$ was constructed so that $\mathbf{P}$ was

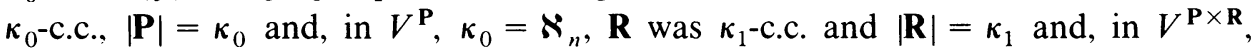
$\kappa_{0}=\boldsymbol{\aleph}_{n}$ and $\kappa_{1}=\boldsymbol{\aleph}_{n+1}$; finally $S$ was $\kappa_{1}$-closed in $V^{\mathbf{P} * \mathbf{R}}, S$ was $\kappa_{2}$-c.c., $|S|=\kappa_{2}$ and, in $V^{\mathbf{P} * \mathbf{R} * S}, \kappa_{0}=\boldsymbol{\aleph}_{n}, \kappa_{1}=\boldsymbol{\aleph}_{n+1}, \kappa_{2}=\boldsymbol{\aleph}_{n+2}$.

Further there was a mastercondition $q \in j(\mathbf{P} * \mathbf{R} * S)$ that guaranteed that if $\hat{G} \subseteq j(\mathbf{P} * \mathbf{R} * S)$ was generic and $q \in \hat{G}$ then there is a $G \subseteq \mathbf{P} * \mathbf{R} * S$ such that $j$ can be extended to $\hat{j}: V[G] \rightarrow M[\hat{G}]$.

We consider the ultrafilter generated by $\hat{j}$ and $j^{\prime \prime} \kappa_{1}$. This is an ultrafilter $U^{*}$ on $\left[\kappa_{1}\right]^{\kappa_{0}}$. Since $\hat{G} \subseteq j(\mathbf{P} * \mathbf{R} * S), \hat{G}=\hat{G}_{0} * \hat{G}_{1} * \hat{G}_{2}$ where $\hat{G}_{0} * \hat{G}_{1} \subseteq j(\mathbf{P} * \mathbf{R})$ and $\hat{G}_{2} \subseteq$ $j(S)$. By assumption $j(S)$ is $<\kappa_{2}$-closed in $V\left[\hat{G}_{0} * \hat{G}_{1}\right]$. Since $\mathscr{P}\left(\left[\kappa_{1}\right]^{\kappa_{0}}\right)^{V[G]}$ has cardinality $\kappa_{2}$ we can apply Lemma 18 to get a normal, fine, $\kappa_{0}$-complete ultrafilter $U^{*}$ on $\mathscr{P}\left(\left[\kappa_{1}\right]^{\kappa_{0}}\right)^{V[G]}$ inside $V\left[\hat{G}_{0} * \hat{G}_{1}\right]$. In $V[G]$, let $\mathscr{I}=\left\{x \subseteq\left[\kappa_{1}\right]^{\kappa_{0}}\right.$ : $\left.\left\|x \notin U^{*}\right\|_{\hat{G}_{0} * \hat{G}_{1} / G}=1\right\}$. Since $j(\mathbf{P} * \mathbf{R})$ is $\kappa_{2}$-c.c. Lemma 17 implies that $\mathscr{I}$ is $\kappa_{2}$-saturated. Hence in this model there is a normal, fine, $\boldsymbol{\kappa}_{n}$-complete $\boldsymbol{\kappa}_{n+2}$-saturated ideal on $\left[\boldsymbol{\aleph}_{n+1}\right]^{\aleph_{n}}$. Thanks to Magidor's Proposition 9 we know this ideal is precipitous. (We could argue this directly too.) We sum up Examples 5 and 6 by the following theorem.

THEOREM. If ZFC + there is a 2-huge cardinal is consistent then so is ZFC+

(a) there is an $\boldsymbol{\aleph}_{n}$-complete, normal fine precipitous ideal on $\left[\boldsymbol{\aleph}_{n+2}\right]^{\boldsymbol{\aleph}_{n+1}}$,

(b) there is a normal, fine, precipitous $\boldsymbol{\aleph}_{n}$-complete, $\boldsymbol{\aleph}_{n+2}$-saturated ideal on $\left[\boldsymbol{\aleph}_{n+1}\right]^{\boldsymbol{N}_{n}}$.

Note. For (b) an almost-two-huge cardinal suffices.

It is shown in [F-M2] that, from a huge cardinal, it is consistent that there is an $\aleph_{n+1}$-saturated ideal on $\boldsymbol{\aleph}_{\omega_{n+1}}$. This shows that an impossibility result of Kunen is sharp.

We will now honor an obligation from earlier in the paper and prove the consistency of the weak Resemblance Axiom. In [F1] the author proved the following theorem:

TheOREM. ZFC + there is a huge cardinal implies Con $(Z F C+$ G.C.H. + every regular cardinal $\kappa$ carries $a \kappa^{+}$-saturated ideal ).

We now show the following theorem.

THEOREM 19. If G.C.H. holds and for all $n, \boldsymbol{\aleph}_{n}$ carries an $\boldsymbol{\aleph}_{n+1}$-saturated ideal, then the weak Axiom of Resemblance holds.

In fact we can show, using these techniques, that if the G.C.H. holds and every regular cardinal carries a $\kappa^{+}$-saturated ideal then for all sequences $\kappa_{1}<\cdots<\kappa_{n}$ and $\lambda_{1}<\cdots<\lambda_{n}$ of uncountable regular cardinals such that $\kappa_{i} \leqslant \lambda_{i}$ there is a generic elementary embedding $j: V \rightarrow M$ such that $j\left(\kappa_{i}\right)=\lambda_{i}$.

We do not need the G.C.H. assumption if the ideal on each $\kappa$ is $\kappa$-centered. This is possible also, using the techniques of [F-L]. 
Proof OF Theorem 19. Let $\mathscr{I}$ be an $\aleph_{n+1}$-saturated ideal on $\aleph_{n}$. Let $\kappa$ be a regular cardinal $>\boldsymbol{\aleph}_{n+1}$. Let $\alpha<\kappa$, and $j_{0, \alpha}$ be the embedding of $V \rightarrow M_{\alpha}$ where $j_{0, \alpha}$ and $M_{\alpha}$ are the results of iterating $\mathscr{I} \alpha$-times.

Claim. (a) $j_{0 . \alpha}(\kappa)=\kappa$,

(b) $j_{0, \kappa}\left(\aleph_{n}\right)=\kappa$.

Proof. First we note that

$$
\left|\mathscr{P}_{f}\left(\boldsymbol{\aleph}_{n}^{\alpha}\right)\right|=2^{\aleph_{n}} \times \alpha=\boldsymbol{\aleph}_{n+1} \cdot \alpha<\kappa .
$$

Hence $\mathbf{P}_{\alpha}=\mathscr{P}_{f}\left(\boldsymbol{\aleph}_{n}^{\alpha}\right) / \mathscr{I}^{\alpha}$ has the $\kappa$-c.c. Thus $V^{\mathbf{P}_{\alpha}} \vDash \kappa$ is regular.

In $M_{\alpha}, j_{0, \alpha}(\kappa)=\left\{[f]_{M_{\alpha}} \mid f: \boldsymbol{\aleph}_{n}^{\alpha} \rightarrow \kappa\right.$ and $f$ has finite supports $\}$. An $f:\left(\boldsymbol{\aleph}_{n}\right)^{\alpha} \rightarrow \kappa$ with finite support is bounded in $\kappa$. The number of functions bounded by any $\gamma<\kappa$ is $\gamma^{\kappa_{n}} \times \alpha<\kappa$. Hence in $V^{\mathbf{P}_{a}},\left|j_{0, \alpha}(\gamma)\right|<\kappa$. Since $\left\{j_{0 . \alpha}(\gamma): \gamma<\kappa\right\}$ is cofinal in $j_{0 . \alpha}(\kappa)$ we get that $j_{0, \alpha}(\kappa)=\kappa$.

To see (b) we note that $j_{0, \alpha}\left(\boldsymbol{\aleph}_{n}\right)$ is a proper end extension of $j_{0, \beta}\left(\boldsymbol{\aleph}_{n}\right)$ if $\beta<\alpha$. Hence $j_{0 . \kappa}\left(\boldsymbol{\aleph}_{n}\right)=\bigcup_{\alpha<\kappa} j_{0 . \alpha}\left(\boldsymbol{\aleph}_{n}\right) \geqslant \kappa$. On the other hand for $\alpha<\kappa, j_{0 . \alpha}\left(\boldsymbol{\aleph}_{n}\right)<\kappa$, hence $\bigcup_{\alpha<\kappa} i_{0, \alpha}\left(\aleph_{n}\right)=\kappa$.

To prove the theorem we repeatedly use the claim. We prove by induction on $n$ that, if $\kappa_{1}<\kappa_{2}<\cdots<\kappa_{n}$, there is a generic embedding $j: V \rightarrow M$ such that $j\left(\boldsymbol{\aleph}_{i}\right)=\kappa_{i}$ and the forcing for doing this has cardinality $\leqslant \kappa_{n}$.

For $n=1$.

Case 1. $\kappa_{1}=\boldsymbol{\kappa}_{2}$.

Let $j_{0,1}$ be the embedding gotten by forcing with the $\boldsymbol{\aleph}_{2}$-saturated ideal on $\boldsymbol{\aleph}_{1}$. Then, $j_{0,1}\left(\boldsymbol{\aleph}_{1}\right) \leqslant \boldsymbol{\aleph}_{2}$, since $\boldsymbol{\kappa}_{2}$ is preserved by the forcing. On the other hand, since $M$ is closed under countable sequences in $V^{\mathbf{P}}, j_{0,1}\left(\boldsymbol{\aleph}_{1}\right) \geqslant \boldsymbol{\aleph}_{2}$. Hence, $j_{0,1}\left(\boldsymbol{\aleph}_{1}\right)=\boldsymbol{\aleph}_{2}$.

Case 2. $\kappa_{1}>\boldsymbol{\kappa}_{2}$.

By the claim $j_{0, \kappa_{1}}\left(\aleph_{1}\right)=\kappa_{1}$ where $j_{0, \kappa_{1}}$ is the result of iterating the ideal on $\boldsymbol{\aleph}_{1}$ $\kappa_{1}$-times.

For $n+1$. Suppose $\kappa_{1}<\cdots<\kappa_{n}<\kappa_{n+1}$ are regular cardinals. By our induction hypothesis there is a forcing $\mathbf{P},|\mathbf{P}| \leqslant \kappa_{n}$ and in $V^{\mathbf{P}}$ there is an elementary embedding $j: V \rightarrow M$ such that, for all $i \leqslant n, j\left(\boldsymbol{\aleph}_{i}\right)=\kappa_{i}$. Since $|\mathbf{P}| \leqslant \kappa_{n}, \mathbf{P}$ has the $\kappa_{n+1}$-c.c. Hence $\kappa_{n+1}$ is regular in $V^{\mathbf{P}}$ and so is regular in $M$. Since $M \vDash \kappa_{i}=\boldsymbol{\aleph}_{i}$, $M \vDash \kappa$ is a regular cardinal $\geqslant \boldsymbol{\aleph}_{n+1}$ and $\boldsymbol{\aleph}_{n+1}^{M}=j\left(\boldsymbol{\aleph}_{n+1}^{V}\right)$. Hence, in $M$ there is an $\aleph_{n+2}$-saturated ideal $\mathscr{I}$ on $\aleph_{n+1}$. Arguing as for the case $n=1$, we find a partial ordering $Q \in M$ for iterating $\mathscr{I}$ such that $|Q|^{M}=\kappa_{n+1}$, and forcing with $Q$ we get an embedding $k: M \rightarrow N$ with critical point $\aleph_{n+1}^{M}$ such that $k\left(\aleph_{n+1}^{M}\right)=\kappa_{n+1}$. Forcing with $\mathbf{P} * Q$ then gives us an embedding $k \circ j: V \rightarrow N$ such that, for all $1 \leqslant i \leqslant n+1, h \circ j\left(\boldsymbol{\aleph}_{i}\right)=\kappa_{i}$.

4. In this section we show that some of these generic axioms imply the G.C.H. Along the way we must take a detour through graph theory.

Definition. Say that $[\kappa, \lambda] \rightarrow\left[\kappa^{\prime}, \lambda^{\prime}\right]$ iff every graph of cardinality $\kappa$ and chromatic number $\lambda$ has a subgraph of cardinality $\kappa^{\prime}$ and chromatic number $\lambda^{\prime}$. (Here we use the term subgraph for an induced subgraph.) 
In [Ga] Galvin conjectured that for every graph $G$ of chromatic number $\kappa$ and every $\lambda<\kappa$ there is a subgraph of $G$ of chromatic number $\lambda$. In the same paper he proved some negative results which we will cite and use below.

The following consistency results are known.

THEOREM [B] (G.C.H) (BAuMgARTNER). There is a countably closed, $\boldsymbol{\aleph}_{2}$-c.c. partial ordering $\mathbf{P}$ such that, in $V^{\mathbf{P}},\left[\boldsymbol{\aleph}_{2}, \boldsymbol{N}_{2}\right] \nrightarrow\left[\boldsymbol{N}_{1}, \boldsymbol{\aleph}_{1}\right]$.

THEOREM [F-L] (Foreman, LAVER). If ZFC + there is a huge cardinal is consistent, then for any $n>k \in \omega$ so is $Z F C+\left[\boldsymbol{\aleph}_{n}, \boldsymbol{\aleph}_{n}\right] \rightarrow\left[\boldsymbol{\aleph}_{k}, \boldsymbol{\aleph}_{k}\right]$.

We now show that these ideals imply many of the graph transfer results.

LEMma 20. Suppose that there is a precipitous ideal on $[\kappa]^{\kappa^{\prime}}$ concentrating on $[\lambda]^{\lambda^{\prime}}$ that is $\gamma$-centered for some $\gamma<\lambda$. Then $[\kappa, \lambda] \rightarrow\left[\kappa^{\prime}, \lambda^{\prime}\right]$.

Proof. Let $j: V \rightarrow M$ be the elementary embedding induced by the ideal. Let $H$ be a graph on $\kappa$ with chromatic number $\lambda^{-}$. Suppose $H$ has no subgraph of cardinality $\kappa^{\prime}$ and chromatic number $\lambda^{\prime}$. Consider $j^{\prime \prime} H \subseteq j(H)$.

By Lemma $5, j^{\prime \prime} H \in M$ and $j^{\prime \prime} H$ has order type $\kappa=j\left(\kappa^{\prime}\right)$ and $j\left(\lambda^{\prime}\right)=\lambda$. Since $M$ thinks that $j(H)$ is a counterexample to $[j(\kappa), j(\lambda)] \rightarrow\left[j\left(\kappa^{\prime}\right), j\left(\lambda^{\prime}\right)\right]$, and $j^{\prime \prime} H \simeq H, M \vDash \operatorname{chrom}(H)<j\left(\lambda^{\prime}\right)=\lambda$. Hence if $G \subseteq \mathbf{P}=\mathscr{P}\left([\kappa]^{\kappa^{\prime}}\right) / \mathscr{I}$ is generic then $V[G] \vDash \operatorname{chrom}(H)<\lambda$.

Let $\tau \in V^{\mathbf{P}}$ be a term for a coloring of $H$ into $\delta$-colors where $\delta<\lambda$. Let $\left\langle\mathscr{F}_{\alpha}\right.$ : $\alpha<\gamma\rangle$ be a collection of filters so that $\mathbf{P}=\cup_{\alpha<\gamma} \mathscr{F}_{\alpha}$. Define a coloring $c$ of $H$ into $\gamma \times \delta$ many colors by $c(h)=(\alpha, \beta)$ where, for some $p \in \mathscr{F}_{\alpha}, p \Vdash \tau(h)=\beta$. To see that this is a coloring we note that if $c(h)=c\left(h^{\prime}\right)=(\alpha, \beta)$ then there are $p$, $p^{\prime} \in \mathscr{F}_{\alpha}, p \Vdash \tau(h)=\beta$ and $p^{\prime} \Vdash \tau\left(h^{\prime}\right)=\beta$. Since $p$ and $p^{\prime}$ are compatible, $h$ and $h^{\prime}$ are not connected in $H$.

Thus in $V$, $\operatorname{chrom}(H)<\lambda$. This is a contradiction.

A theorem of Galvin [Ga] now gives us our first results on the behavior of the continuum.

THEOREM (GALVIN). Suppose that $2^{\aleph_{n}}=2^{\aleph_{n+1}}<2^{\aleph_{n+2}}$, then there is a graph on $\left(2^{\aleph_{n+1}}\right)^{+}$of chromatic number $\boldsymbol{\aleph}_{n+2}$ with no subgraph of chromatic number $\boldsymbol{\aleph}_{n+1}$.

We deduce some corollaries from Lemma 20 and Galvin's theorem.

Corollary 21. Suppose there is an $\aleph_{n+1}$-centered ideal $\mathscr{I}$ on $\left[\left(2^{\aleph_{n+1}}\right)^{+}\right]^{2^{\aleph_{n+1}}}$ then $2^{\aleph_{n}}<2^{\aleph_{n+1}}$.

We will prove the corollary by deriving a contradiction.

Proof. We first show that, for some $m \geqslant n \in \omega, 2^{\aleph_{n}}<2^{\aleph_{m}}$. Otherwise for all $m \geqslant n, 2^{\aleph_{m}} \geqslant \aleph_{\omega+1}$. But, by the corollary to Magidor's theorem, this cannot happen. (Our original proof did not make use of Magidor's theorem.)

Hence we know that $2^{\aleph_{n+1}}<\aleph_{\omega}$ and that there is an $m \geqslant n$ such that $2^{\aleph_{m+1}}=$ $2^{\aleph_{n+1}}$ and $2^{\aleph_{m+2}}>2^{\boldsymbol{\aleph}_{m+1}}$. Since $\mathscr{I}$ is $\boldsymbol{\aleph}_{n+1}$-centered it is $\boldsymbol{\aleph}_{m+1}$-centered. Thus without loss of generality $2^{\aleph_{n+1}}<2^{\aleph_{n+2}}$. By Galvin's result, if $2^{\aleph_{n}}=2^{\aleph_{n+1}}$ there is a 
graph $H$ on $\left(2^{\boldsymbol{N}_{n+1}}\right)^{+}$of chromatic number $\boldsymbol{\aleph}_{n+2}$ with no subgraph of chromatic number $\boldsymbol{\aleph}_{n+1}$. By Remark 13 we know that $\mathscr{I}$ concentrates on $\left[\boldsymbol{\kappa}_{n+2}\right]^{\boldsymbol{N}_{n+1}}$. Hence by Lemma 20, $H$ has a subgraph of chromatic number $\aleph_{n+1}$, a contradiction.

We now use a theorem of Baumgartner's.

Theorem (BAumgartner). Suppose $2^{\kappa_{0}}<2^{\aleph_{1}}$ and $2^{\aleph_{0}}<\boldsymbol{\aleph}_{\omega}$, then there is an almost disjoint family of functions from $\omega_{1}$ to $\omega_{1}$ of size $2^{\omega_{1}}$.

The following corollary is well known:

COROllary. If there is an $\boldsymbol{\aleph}_{2}$-saturated ideal on $\boldsymbol{\aleph}_{1}$ and $\omega_{1}<2^{\boldsymbol{\aleph}_{0}}<\boldsymbol{\aleph}_{\omega}$, then $2^{\kappa_{0}}=2^{\kappa_{1}}$.

We now use these results to prove a theorem that settles the G.C.H. below $\boldsymbol{\aleph}_{\omega}$ assuming the existence of a particular generic large cardinal embedding.

Theorem 22. Suppose there is a partial ordering $\mathbf{P}$ that is $\boldsymbol{\aleph}_{1}$-centered and in $V^{\mathbf{P}}$ there is an elementary embedding $j: V \rightarrow M$ such that $j^{\prime \prime} \boldsymbol{\aleph}_{\omega} \times 2^{\boldsymbol{\kappa}_{0}} \in M$ and $\operatorname{crit}(j)=$ $\omega_{1}$. Then the G.C.H. holds below $\boldsymbol{\aleph}_{\omega}$.

Proof. We first argue that $2^{\boldsymbol{\aleph}_{0}}<\boldsymbol{\aleph}_{\omega}$. Let $\lambda=2^{\boldsymbol{\aleph}_{0}} \times \boldsymbol{\aleph}_{\omega}$. In $V^{\mathbf{P}}$ we have an ultrafilter on $\left([\lambda]^{\leqslant \lambda}\right)^{V}$ induced by $j$ and $j^{\prime \prime} \lambda$. Hence in $V$ we have an $\boldsymbol{\aleph}_{1}$-centered ideal on $[\lambda] \leqslant \lambda$. Thus, by the corollary to Magidor's theorem $\lambda \leqslant \boldsymbol{\aleph}_{\omega}$. Hence $2^{\aleph_{0}}<\boldsymbol{\aleph}_{\omega}$.

Without loss of generality, $j: V \rightarrow M$ is the embedding induced by the ideal $\mathscr{I}$.

We claim that, for all $n>0, j\left(\boldsymbol{\aleph}_{n}\right)=\boldsymbol{\aleph}_{n+1}$. Otherwise, let $n$ be the least counterexample. Since $\mathscr{P}\left(\boldsymbol{\aleph}_{n}\right)^{V} \subseteq M$ and $M \vDash j\left(\boldsymbol{\aleph}_{n}^{V}\right)$ is a cardinal, $j\left(\boldsymbol{\aleph}_{n}^{V}\right) \geqslant \boldsymbol{\aleph}_{n+1}^{V}$. On the other hand, if $n>1, j\left(\boldsymbol{\aleph}_{n-1}^{V}\right)=\boldsymbol{\aleph}_{n}^{V}$ and $M \vDash j\left(\boldsymbol{\aleph}_{n}\right)$ is the successor of $j\left(\boldsymbol{\aleph}_{n-1}\right)$. Hence if $\kappa=\boldsymbol{\aleph}_{n}^{V}, M \vDash j\left(\boldsymbol{\aleph}_{n}\right) \leqslant \boldsymbol{\kappa}^{+}$. By the saturation of $\mathscr{I}, \boldsymbol{\aleph}_{n+1}^{V}$ is a cardinal in $M$, hence $j\left(\boldsymbol{\aleph}_{n}^{V}\right)=\boldsymbol{\aleph}_{n+1}^{V}$. The case $n=1$ is similar.

We now show that if $2^{\boldsymbol{\aleph}_{0}}=\boldsymbol{\aleph}_{k}$ then, for all $n, 2^{\boldsymbol{\aleph}_{n}} \leqslant \boldsymbol{\aleph}_{n+k}$ : let $k$ be such that $2^{\boldsymbol{\aleph}_{0}}=\boldsymbol{\aleph}_{k}$. If $2^{\boldsymbol{N}_{n}} \geqslant \boldsymbol{\aleph}_{n+k+1}$, let $\left\langle x_{\alpha}: \alpha<\boldsymbol{\aleph}_{n+k+1}\right\rangle$ be a sequence of distinct elements of $\left[\boldsymbol{\aleph}_{n+1}\right]^{<\boldsymbol{\aleph}_{n+1}}$ of length $\boldsymbol{\aleph}_{n+k+1}$. Let $M_{n}$ be the result of iterating $\mathscr{I} n$-times. Then if $j_{n}: V \rightarrow M_{n}$ is the embedding induced by this iteration, $j_{n}\left(\boldsymbol{\aleph}_{1}\right)=\boldsymbol{\aleph}_{n+1}^{V}$ and $\left\langle x_{\alpha}: \alpha\left\langle\boldsymbol{\aleph}_{n+k+1}^{V}\right\rangle \in M_{n}\right.$. By our earlier remarks $\boldsymbol{\aleph}_{n+k+1}^{V}=\boldsymbol{\aleph}_{k+1}^{M_{n}}$. Hence $M_{n} \vDash\left\langle x_{\alpha}\right.$ : $\alpha\left\langle\boldsymbol{\aleph}_{n+k+1}^{V}\right\rangle$ is a sequence of elements of $\left[\boldsymbol{\aleph}_{1}\right]^{<\boldsymbol{\aleph}_{1}}$ of length $\boldsymbol{\aleph}_{k+1}^{M}$. However, by the elementarity of $j_{n}, M_{n} \vDash 2^{\aleph_{0}}=\boldsymbol{\aleph}_{k}$, a contradiction.

Suppose that $2^{\boldsymbol{\kappa}_{0}}=2^{\boldsymbol{\kappa}_{1}}$. Choose the least $n$ such that $2^{\boldsymbol{\aleph}_{0}}<2^{\boldsymbol{\kappa}_{n+2}}$. Since $2^{\boldsymbol{\aleph}_{n+1}}<$ $\aleph_{\omega}, j\left(2^{\aleph_{n+1}}\right)=\left(\left(2^{\aleph_{n+1}}\right)^{+}\right)^{V}$, so $\mathscr{I}$ concentrates on $\left[\left(2^{\aleph_{n+1}}\right)^{+}\right]^{2^{\kappa_{n+1}}}$. Projecting $\mathscr{I}$ to an ideal $J$ on $\left[\left(2^{\aleph_{n+1}}\right)^{+}\right]^{2^{\kappa_{n+1}}}$ by Lemma 4 , the ideal $J$ is $\aleph_{1}$-centered. Hence we get that $2^{\aleph_{n}}<2^{\kappa_{n}+1}$ contradicting our choice of $n$. Hence $2^{\kappa_{0}}<2^{\kappa_{1}}$.

Now we project our ideal on $\boldsymbol{\aleph}_{1}$ by the map $\pi$ : $\left[\boldsymbol{\kappa}_{\omega}\right]^{\boldsymbol{\kappa}_{\omega}} \rightarrow \boldsymbol{\kappa}_{1}$ defined by $\pi(x)=\sup x \cap \omega_{1}$. Then $\pi(\mathscr{I})$ is an $\boldsymbol{\aleph}_{1}$-centered ideal on $\boldsymbol{\aleph}_{1}$. Hence by the corollary to Baumgartner's theorem $2^{\kappa_{0}}=\aleph_{1}$. Thus by previous arguments, for all $n, 2^{\aleph_{n}}=\boldsymbol{\aleph}_{n+1}$. 
Assuming the Axiom of Resemblance and the existence of am embedding satisfying the hypothesis of Theorem 22, we can deduce the G.C.H. in the Universe. Thus adoption of these ideal axioms settle the G.C.H. Unfortunately, as we will see, certain other ideal axioms imply that $2^{\boldsymbol{N}_{0}} \geqslant \boldsymbol{N}_{2}$.

We finish our excursion into graph theory by giving a partial answer to a question Erdös asked the author. Erdös referred to the question as "Taylor's problem":

TAYlor's Problem. Suppose that $G$ is a graph on $\kappa$ of chromatic number $\kappa$ and $\lambda>\kappa$. Is there a graph $H$ on $\lambda$ with chromatic number $\lambda$ such that $H$ has the same finite subgraphs that $G$ does?

We must explicate the notion of subgraph for which we can get results. If $G$ and $H$ are graphs, we say that $G$ is a weak subgraph of $H$ iff the nodes of $G$ are a subset of the nodes of $H$ and the edges of $G$ are a subset of the edges of $H$.

THEOREM 23. Suppose that $2^{\kappa}=\kappa^{+}$and that there is a countably complete, $\kappa$-centered ideal $\mathscr{I}$ on $\left[\kappa^{+n+1}\right]^{\kappa^{+n}}$. Then if $1 \leqslant m<p \leqslant n+1$ and $G$ is a graph on $\kappa^{+m}$ of chromatic number $\kappa^{+m}$ then there is a graph $H$ on $\kappa^{+p}$ of chromatic number $\kappa^{+p}$ such that $G$ and $H$ have the same finite weak subgraphs.

Proof. It is clearly enough to see this for the case $p=m+1$. By Magidor's Proposition $9, \mathscr{I}$ is precipitous. Consider $j(G), j(G)$ is a graph on $\kappa^{+m+1}$ of chromatic number $\kappa^{+m+1}$ in $M$. Let $\mathbf{P}=\mathscr{P}\left(\left[\kappa^{+n+1}\right]^{\kappa^{+n}}\right) / \mathscr{I}$ and let $\mathscr{F} \subseteq \mathbf{P}$ be generic. Since $M$ is closed under $\kappa^{+n+1}$-sequences in $V[\mathscr{F}], j(G)$ has chromatic number $\kappa^{+m+1}$ in $V[\mathscr{F}]$. In $V[\mathscr{F}]$ let $G^{*}$ be an isomorphic copy of $j(G)$ and $f$ : $j(G) \rightarrow G^{*}$ be an isomorphism so that $f \circ j: G \rightarrow G^{*}$ is the identity.

Let $\left\langle\mathscr{F}_{\alpha}: \alpha<\kappa\right\rangle$ be centering of P. For each $\alpha<\kappa$ let $G_{\alpha}$ be the graph on $\kappa^{+m+1}$ defined by putting an edge between $\gamma$ and $\delta$ iff there is a $p \in \mathscr{F}_{\alpha}$

$$
p \Vdash \gamma \text { and } \delta \text { have an edge between them in } G^{*} .
$$

We claim that each $G_{\alpha}$ and $G$ have the same finite weak subgraphs. Since $G^{*} \uparrow \kappa^{+m}$ is determined to be $G$ with Boolean value $1, G$ is a subgraph of every $G_{\alpha}$. Consequently, \{weak subgraphs of $G\} \subseteq\left\{\right.$ weak subgraph of $G_{\alpha}$ \}. On the other hand, if $K \subseteq G_{\alpha}$ is a finite weak subgraph then there is a $p \in \mathscr{F}_{\alpha}$ such that for all $\gamma$, $\delta \in K$ if $\gamma$ and $\delta$ are connected in $K, p \Vdash \gamma$ and $\delta$ are connected. Hence $p \Vdash K$ is a weak subgraph of $G^{*}$ and hence of $j(G)$. However, since $j$ is elementary, $j(G)$ and $G$ have the same weak subgraphs. Thus $K$ is a weak subgraph of $G$.

We now need to show that, for some $\alpha, \operatorname{chrom}\left(G_{\alpha}\right)=\kappa^{+m+1}$. Otherwise for each $\alpha$ let $c_{\alpha}: G_{\alpha} \rightarrow \kappa^{+m}$ be a coloring. Let $\mathscr{C}: \kappa^{+m+1} \rightarrow\left(\kappa^{+m}\right)^{\kappa}$ be defined by

$$
\mathscr{C}(\gamma)=\left\langle c_{\alpha}(\gamma): \alpha<\kappa\right\rangle
$$

Since $\left(\kappa^{+m}\right)^{\kappa}=\kappa^{+m}$ we will be done if we can show that $\mathscr{C}$ is a coloring of $G^{*}$ in $V[\mathscr{F}]$, since this contradicts $\operatorname{chrom}(j(G))=\kappa^{+m+1}$. Let $\gamma, \delta \in \kappa^{+m+1}$. Suppose $\gamma$, $\delta$ are connected in $j(G)$, then there is a $p \in \mathscr{F}, p \Vdash \gamma$ and $\delta$ are connected in $G^{*}$. However, $p \in \mathscr{F}_{\alpha}$ for some $\alpha$. Hence $\gamma$ and $\delta$ are connected in $G_{\alpha}$. But then $c_{\alpha}(\gamma) \neq c_{\alpha}(\delta)$ and hence $\mathscr{C}(\gamma) \neq \mathscr{C}(\delta)$. 
The proof above actually gives more information. Namely, under the same hypothesis, it shows that if $1 \leqslant r \leqslant m \leqslant n$ and $G$ is a graph on $\kappa^{+m}$ with chromatic number $\kappa^{+r}$, then there is a graph $G$ on $\kappa^{+m+1}$ with chromatic number $\kappa^{+r+1}$ such that $G$ and $H$ have the same finite weak subgraphs.

5. We now turn our attention to Lebesgue measure. Two classical theorems motivate our discussion on this

THEOREM (MYCIELSKI - SWIERCZKOWSKI) [M-S]. The axiom of determinacy implies that every set of reals is Lebesgue measurable.

THEOREM (SOlOVAY) [So1]. If ZFC + there is an inaccessible cardinal is consistent then so is $Z F C+$ every set of reals in $L(\mathbf{R})$ is Lebesgue measurable.

Ideals have been known for some time to have an effect on Lebesgue measure.

For example Magidor in [M1] showed the following

THEOREM. If the C.H. holds, there is a measurable cardinal and a precipitous ideal on $\omega_{1}$, then every $\Sigma_{3}^{\prime}$ set of reals is Lebesgue measurable.

Magidor got a similar result for $\Sigma_{4}^{\prime}$ assuming a precipitous ideal on $\left[2^{2 \omega_{1}}\right]^{<\omega_{1}}$. Woodin in [W2] showed the following

THEOREM. If the C.H. holds and there is an $\boldsymbol{\aleph}_{1}$-dense ideal on $\boldsymbol{\aleph}_{1}$, then every set of reals in $L(\mathbf{R})$ is Lebesgue measurable.

We now prove the following theorem.

THEOREM 24. If there is a $2^{\boldsymbol{N}_{1}}$-dense ideal $\mathscr{I}$ on $\left[\left(2^{\boldsymbol{N}_{1}}\right)^{+}\right]^{\boldsymbol{N}_{1}}$ then every set of reals in $L(\mathbf{R})$ :

(a) is Lebesgue measurable,

(b) has the property of Baire.

Further, in $L(\mathbf{R}), \omega \rightarrow(\omega)^{\omega}$.

To prove this we use a lemma of Solovay from [So1].

Lemma. Let $\phi$ be a formula, $\vec{\alpha}$ a finite sequence of ordinals and $r$ a real. Let $\mathbf{P}=\operatorname{Col}\left(\omega, 2^{\boldsymbol{N}_{1}}\right]$. In $V^{\mathbf{P}},\{x \in \mathbf{R}: L(\mathbf{R}) \Vdash \phi(x, r, \vec{\alpha})\}$ is Lebesgue measurable.

Mathias [Ma] proved the following

Lemma. Suppose $\phi$ is a formula, $\vec{\alpha}$ a finite set of ordinals and $r$ a real. Let $\mathbf{P}=\operatorname{Col}\left(\omega, 2^{\alpha_{0}}\right]$. Then if $L(\mathbf{R}) \vDash \phi(x, r, \vec{\alpha})$ defines a partition $f:[\omega]^{\omega} \rightarrow 2$, then in $V^{\mathbf{P}}$ there is a homogeneous set for $f$.

We also use the following well-known lemma.

Lemma. Suppose $\mathbf{P}$ is a partial ordering such that, for some $\kappa \geqslant \omega_{1},|\mathbf{P}|=\kappa$ and $V^{\mathbf{P}} \vDash \kappa$ is countable. Then $\mathscr{B}(\mathbf{P}) \simeq \mathscr{B}(\operatorname{col}(\omega, \kappa])$. 
Proof of Theorem 24. By Proposition 9 and Lemma $10 \mathscr{I}$ is precipitous.

Let $j: V \rightarrow M$ be the elementary embedding induced by $\mathscr{I}$ and $G$ and generic ultrafilter on $\left[\left(2^{\aleph_{0}}\right)^{+}\right]^{\aleph_{1}}$. Then $j\left(\boldsymbol{\aleph}_{1}\right)=\left(2^{\aleph_{0}}\right)^{+V}$. Hence $\left(2^{\aleph_{0}}\right)^{V}$ is countable in $V[G]$. By assumption, $\mathbf{P}=\mathscr{P}\left(\left[\left(2^{\kappa_{0}}\right)^{+}\right]^{\aleph_{1}}\right) / \mathscr{I}$ has a dense set of cardinality $2^{\boldsymbol{\kappa}_{0}}$, so $\mathbf{P} \sim$ $\operatorname{Col}\left(\omega, 2^{\kappa_{0}}\right]$ by the lemma above.

Let $r \in \mathbf{R}, \vec{\alpha} \in O R^{<\omega}$ and $\phi$ a formula. Then $\phi(x, r, \vec{\alpha})$ defines a set of reals in $L(\mathbf{R})$ by $X=\{x: L(\mathbf{R}) \vDash \phi(x, r, \vec{\alpha})\}$. Further, every set of reals in $L(\mathbf{R})$ can be so defined. We claim that

$M \vDash j(X)$ is Lebesgue measurable and has the property of Baire.

Since $j$ is elementary this implies that $V \vDash X$ is Lebesgue measurable and has the property of Baire.

Since $\mathscr{I}$ has the disjointing property, $M$ is closed under $\left(2^{\boldsymbol{\kappa}_{0}}\right)^{+}$-sequences in $V[G]$. In particular, $L(\mathbf{R})^{M}=L(\mathbf{R})^{V[G]}$. Now, $j(X)=\left\{x \in L(\mathbf{R})^{M}: \quad L(\mathbf{R}) \vDash\right.$ $\phi(x, j(\vec{\alpha}), j(r))\}$. Since $r \in \mathbf{R}, j(r)=r$. Hence $j(X)$ is a set in $L(\mathbf{R})^{V[G]}$ definable from some ordinals and a real parameter in $V$. Thus by Solovay's lemma, $V[G] \vDash$ $j(X)$ is Lebesgue measurable and has the property of Baire. A set being Lebesgue measurable is absolute between $V[G]$ and $L(\mathbf{R})^{V[G]}$. Since $L(\mathbf{R})^{V[G]}=L(\mathbf{R})^{M}$, $M \vDash j(X)$ is Lebesgue measurable and has the Baire property.

To see that $\omega \rightarrow(\omega)^{\omega}$ in $L(\mathbf{R})$ we use a similar argument.

Using recent remarks of Woodin, slightly weaker things suffice here. For example, we can get the same conclusion by assuming a precipitous ideal on $\left[\left(2^{\kappa_{0}}\right)^{+}\right]^{\aleph_{1}}$ such that

$$
\mathscr{B}=\mathscr{P}\left(\left[\left(2^{\aleph_{0}}\right)^{+}\right]^{\aleph_{1}}\right) / \mathscr{I}=\bigcup_{\alpha<\left(2^{\aleph_{0}}\right)^{+}} \mathscr{B}_{\alpha}
$$

where $\left|\mathscr{B}_{\alpha}\right| \leqslant 2^{\aleph_{0}}$ and, for a stationary set of $\alpha<\left(2 \kappa^{\kappa_{0}}\right), \mathscr{B}_{\alpha}$ is a neat subalgebra of $\mathscr{B}$.

Because these axioms imply some consequences of A.D. it is natural to ask whether these axioms imply significant amounts of determinacy. The author conjectures that this is the case. As further evidence for this we can prove

Proposition 25. If there is a $2^{\aleph_{0}}$-dense ideal $\mathscr{I}$ on $\left[\left(2^{\kappa_{0}}\right)^{+}\right]^{\aleph_{1}}$ which projects onto the closed unbounded filter on $\boldsymbol{\aleph}_{1}$, then

(a) there is a countably additive measure $\mu: \mathscr{P}(\{\text { Turing degress }\})^{L(\mathbf{R})} \rightarrow 2$ such that every cone of degrees gets measure one,

(b) there is a countably complete measure $\nu: \mathscr{P}\left(\boldsymbol{\aleph}_{1}\right)^{I(\mathbf{R})} \rightarrow\{0,1\}$.

Proof. Very similar to Theorem 24.

RemarKS. (1) If we have C.H. then we need only an $\boldsymbol{\aleph}_{1}$-dense ideal on $\omega_{1}$.

(2) Properties analogous to those following the proof of Theorem 22 suffice also in this theorem.

(3) Using Proposition 25 Woodin has shown that $L(\mathbf{R}, \nu) \vDash \nu$ is a measure on $\omega_{1}$ where $L(\mathbf{R}, \nu)$ are the sets constructable from $\mathbf{R}$ using $\nu$ as a predicate.

(4) At first glance (a) looks very close to A.D. Woodin has shown that if $\mu=\{x \subseteq\{$ Turing degrees $\}: x$ contains a cone $\}$, then A.D. holds in $L(\mathbf{R})$. 
6. A question in [B-T] is: Is there an $\boldsymbol{\aleph}_{2}$-saturated ideal $\mathscr{I}$ on $\boldsymbol{\aleph}_{1}$ such that whenever $\mathbf{P}$ is a c.c.c. partial ordering, the ideal induced by $\mathscr{I}$ in $V^{\mathbf{P}}$ remains $\boldsymbol{\aleph}_{2}$-saturated? Laver and Baumgartner-Taylor proved the following criterion for remaining $\boldsymbol{\aleph}_{2}$-saturated in $V^{\mathbf{P}}$ :

Let $G \subseteq \mathscr{P}\left(\aleph_{1}\right) / \mathscr{I}$ be generic object. Let $j: V \rightarrow M$ be the elementary embedding induced by $G$.

Saturation CRITERION. $\mathscr{I}$ remains $\boldsymbol{\aleph}_{2}$-saturated in $V^{\mathbf{P}}$ iff $j(\mathbf{P})$ is c.c.c. in $V[G]$.

If $\mathbf{P}$ is a partial ordering that preserves $\boldsymbol{\aleph}_{1}$ and $\boldsymbol{\aleph}_{2}$ and $\overline{\mathscr{I}}$ is an ideal on $\left[\boldsymbol{\aleph}_{2}\right]^{\boldsymbol{\aleph}_{1}}$ then in $V^{\mathbf{P}}$ we can define an ideal $\overline{\mathscr{I}}$ on $\left(\left[\boldsymbol{\aleph}_{2}\right]^{\boldsymbol{\aleph}_{1}}\right)^{V^{\mathbf{P}}}$ as follows: $X \in \overline{\mathscr{I}}$ iff there is a set $Y \in \mathscr{I}$ such that $X \cap\left(\left[\boldsymbol{\aleph}_{2}\right]^{\boldsymbol{\aleph}_{1}}\right)^{V} \subseteq Y$. Let $\mathscr{I}$ be $\boldsymbol{\aleph}_{2}$-saturated, $G_{\mathscr{I}} \subseteq \mathscr{P}\left(\left[\boldsymbol{\aleph}_{2}\right]^{\boldsymbol{\aleph}_{1}}\right) / \mathscr{I}$ generic and $j: V \rightarrow M \subseteq V\left[G_{\mathscr{I}}\right]$ be the induced embedding. Then the proof of the saturation-criterion carries over mutatis-mutandis to prove

\section{Proposition 26. $\| \overline{\mathcal{I}}$ is $\aleph_{2}$-saturated $\|_{\mathbf{P}}=1$ iff $\| j(\mathbf{P})$ is $\boldsymbol{\aleph}_{2}^{V}$-saturated in $V\left[G_{\mathscr{I}}\right] \|=$ 1.}

Corollary 27. If $\mathscr{I}$ is an $\boldsymbol{\aleph}_{2}$-saturated ideal on $\left[\boldsymbol{\aleph}_{2}\right]^{\boldsymbol{\aleph}_{1}}$ then $\mathscr{I}$ remains $\boldsymbol{\aleph}_{2}$ saturated in any c.c.c. extension. In particular, if $J$ is the projection of $\mathscr{I}$ onto $\omega_{1}$ then $J$ remains $\boldsymbol{\aleph}_{2}$-saturated in any c.c.c. forcing extension of $V$.

Proof. The second statement follows directly from the first. Let $\mathbf{P}$ be a c.c.c. partial ordering. In view of the criterion for saturation we must prove that if $G \subseteq \mathscr{P}\left(\left[\boldsymbol{\aleph}_{2}\right]^{\aleph_{1}}\right) / \mathscr{I}$ and $j: V \rightarrow M \subseteq V[G]$ is the induced elementary embedding, then $j(\mathbf{P})$ is $\boldsymbol{\aleph}_{2}^{V}$-c.c. in $V[G]$. But $\boldsymbol{\aleph}_{2}^{V}=\boldsymbol{\aleph}_{1}^{M}=\boldsymbol{\aleph}_{1}^{V[G]}$. Thus we must show that $j(\mathbf{P})$ is c.c.c. in $V[G]$. Since $\mathbf{P}$ is c.c.c. in $V, M \vDash j(\mathbf{P})$ is c.c.c. By Propositions 8 and 10, ${ }^{\kappa_{1}} M \cap V[G] \subseteq M$. Hence if $V[G]$ had an antichain in $j(\mathbf{P})$ of size $\boldsymbol{\kappa}_{1}$ then $M$ would have an antichain in $j(\mathbf{P})$ of size $\boldsymbol{\aleph}_{1}$ contradicting the c.c.c. in $M$.

We now turn our attention to an easy application of these ideals in algebra:

Let $G$ be a group. Say that $G$ is almost-free iff every subgroup $H \subseteq G$ with $|H|<|G|$ is free. It is well known that nonfree, almost-free groups exist in cardinality $\boldsymbol{\aleph}_{n}$ for every $n \in \omega$.

Proposition 28. Suppose there is an $\boldsymbol{\aleph}_{1}$-centered ideal $\mathscr{I}$ on $\left[\boldsymbol{\aleph}_{n}\right]^{\boldsymbol{\kappa}_{n}}{ }^{1}$. Then every almost free group $G$ of cardinality $\boldsymbol{\aleph}_{n}$ can be written as the union of $\boldsymbol{\aleph}_{1}$ subgroups $G=\bigcup_{\alpha<\aleph_{1}} G_{\alpha}$ where $G_{\alpha}$ is a free group.

REMARKS. (a) As the proof will show, this theorem is true in many categories, e.g. abelian groups, modules over a commutative ring, etc.

(b) For $n=2$, this conclusion holds in the model in [F-L].

(c) For abelian groups, Shelah has shown that the conclusion of Proposition 28 for $n=2$ is false in $L$ and consistent relative to a Mahlo cardinal.

Proof of Proposition 28. Let $\mathbf{P}=\mathscr{P}\left(\left[\boldsymbol{\aleph}_{n}\right]^{\boldsymbol{\kappa}_{n-1}}\right) / \mathscr{I}$ be centered by $\left\langle\mathscr{F}_{\alpha}: \alpha\left\langle\boldsymbol{\aleph}_{1}\right\rangle\right.$ where each $\mathscr{F}_{\alpha}$ is a filter. Let $G$ be almost free of cardinality $\boldsymbol{\aleph}_{n}$.

If $H \subseteq \mathbf{P}$ is generic ultrafilter, we claim that $G$ is free in $V[H]$. Let $j: V \rightarrow M$ be the induced elementary embedding, then $j^{\prime \prime} G \in M$ and is a subgroup of $j(G)$ of cardinality $\boldsymbol{\aleph}_{n-1}^{M}$. Hence $M \vDash j^{\prime \prime} G$ is free. Thus $V[H]$ is a model that $j^{\prime \prime} G$ is free (being free is "upwards absolute") and hence that $G$ is free. 
Let $\left\langle\tau_{\beta}: \beta\left\langle\boldsymbol{\aleph}_{n}\right\rangle\right.$ be a term for a free generating set for $G$ in $V[H]$. For each $\alpha<\boldsymbol{\aleph}_{1}$, let $X_{\alpha}=\left\{g \in G\right.$ : there is a $p \in \mathscr{F}_{\alpha}$ and a $\left.\beta<\boldsymbol{\aleph}_{n}, p \Vdash g=\tau_{\beta}\right\}$. We claim that $X_{\alpha}$ freely generates a subgroup $G_{\alpha}$ of $G$. Otherwise there is some nontrivial finite relation $r$, involving $g_{1} \cdots g_{n} \in X_{\alpha}$, that holds in $G$. Since $\mathscr{F}_{\alpha}$ is a filter there is a $p \in \mathscr{F}_{\alpha}$ such that, for some $\beta_{1} \cdots \beta_{n}, p \Vdash g_{i}=\tau_{\beta_{i}}$. But then $p \Vdash$ $r\left(\tau_{\beta_{1}}, \ldots, \tau_{\beta_{n}}\right)=1$. This contradicts $\left\langle\tau_{\beta}: \beta\left\langle\aleph_{n}\right\rangle\right.$ is a free generating set for $G$.

Let $g \in G$. We must show that there is some $\alpha, g \in G_{\alpha}$. (In fact we can prove more-that any finite number of elements of $G$ lie in some $G_{\alpha}$ and each $G_{\alpha}$ is a pure subgroup of $G$.) Since $g \in G$, there is some sequence $\beta_{1} \cdots \beta_{k}, g_{1} \cdots g_{k}$ and a $p$, $p \Vdash \tau_{\beta_{i}}=g_{i}$ and $g$ is in the subgroup generated by $\tau_{\beta_{1}}, \ldots, \tau_{\beta_{k}}$. But $p \in \mathscr{F}_{\alpha}$ for some $\alpha$, so $g \in G_{\alpha}$.

We now develop some technology to show that ideal axioms are not unanimous in their choice of a value for $2^{\kappa_{0}}$. This technology is standard large cardinal theory when dealing with ultrafilters.

Let $\left\langle\kappa_{n}: n \in \omega\right\rangle$ be an increasing sequence of regular cardinals. Let $\pi:\left[\kappa_{n+2}\right]^{\kappa_{n+1}}$ $\rightarrow\left[\kappa_{n+1}\right]^{\kappa_{n}}$ be defined by $\pi(x)=x \cap \kappa_{n+1}$. This produces a map $\Pi: \mathscr{P}\left(\left[\kappa_{n+2}\right]^{\kappa_{n+1}}\right)$ $\rightarrow \mathscr{P}\left(\left[\kappa_{n+1}\right]^{\kappa_{n}}\right)$. A tower of ideals on $\left\langle\kappa_{n}: n \in \omega\right\rangle$ is a sequence of ideals $\left\langle\mathscr{I}_{n}\right.$ : $n \in \omega\rangle$ where $\mathscr{I}_{n}$ is an ideal on $\left[\kappa_{n+1}\right]^{\kappa_{n}}$ concentrating on $\left[\kappa_{n}\right]^{\kappa_{n-1}}$ and $\Pi_{n+1}\left(\mathscr{I}_{n+1}\right)$ $=\mathscr{I}_{n}$.

Let $\lambda=\sup _{n \in \omega} \kappa_{n}$. A set $X \subseteq[\lambda]^{\lambda}$ is said to have finite support iff, for some $n$, if $x, y \in[\lambda]^{\lambda}$ ard $x \cap \kappa_{n}=y \cap \kappa_{n}$ then $x \in X$ iff $y \in X$. A function $F:[\lambda]^{\lambda} \rightarrow V$ has finite support iff there is an $n$ such that if $x \cap \kappa_{n}=y \cap \kappa_{n}$ then $F(x)=F(y)$. Let $\mathscr{P}_{f}\left([\lambda]^{\lambda}\right)=\left\{X \subseteq[\lambda]^{\lambda}: X\right.$ has finite support $\}$. Then a tower of ideals $\left\langle\mathscr{I}_{n}\right.$ : $n \in \omega\rangle$ defines an ideal on the Boolean algebra $\mathscr{P}_{f}\left([\lambda]^{\lambda}\right)$ by $X \in \mathscr{I}_{\omega}$ iff, for some $n$, $\left\{x \cap \kappa_{n+1}: x \in X\right\} \in \mathscr{I}_{n}$. Since the ideals cohere under the projection maps this is well defined. Given a $V$-ultrafilter $G$ on $\mathscr{P}_{f}\left([\lambda]^{\lambda}\right)$ we can take the ultraproduct of $V$ by functions with finite supports. We get a model $M$ whose universe consists of $G$ equivalence classes of functions $F:[\lambda]^{\lambda} \rightarrow V$ with finite support. It is easy to check that all of the standard ultraproduct theory goes through in this case including Lôs' theorem.

If $G \subseteq \mathscr{P}_{f}\left([\lambda]^{\lambda}\right) / \mathscr{I}_{\omega}$ is a generic ultrafilter we can ask if the ultroproduct by $G$ is well founded. If this is always the case then we say that the tower is precipitous.

Similarly we say that $\mathscr{I}_{\omega}$ is $\kappa$-saturated iff $\mathscr{P}_{f}\left([\lambda]^{\lambda}\right) / \mathscr{I}_{\omega}$ has the $\kappa$ c.c. and $\kappa$-dense iff $\mathscr{P}_{f}\left([\lambda]^{\lambda}\right) / \mathscr{I}$ has a dense subset of cardinality $\kappa$.

Many attempts have been made to establish the consistency of the proposition " $\aleph_{\omega}$ is a Jonsson cardinal" from large cardinals. From Proposition 16, we see that a precipitous ideal on $\left[\boldsymbol{\aleph}_{\omega}\right]^{\boldsymbol{\aleph}_{\omega}}$ implies that $\boldsymbol{\aleph}_{\omega}$ is Jonsson. It is however difficult to conceive of a plan to get a precipitous ideal on $\left[\boldsymbol{\kappa}_{\omega}\right]^{\boldsymbol{\kappa}_{\omega}}$ from large cardinals. The following proposition presents a more reasonable target:

Proposition 29. Let $\left\langle\kappa_{n}: n \in \omega\right\rangle$ be a sequence of regular cardinals and $\lambda=$ $\sup _{n \in \omega} \kappa_{n}$. Suppose there is a precipitous tower $\left\langle\mathscr{I}_{n}: n \in \omega\right\rangle$ on $\left\langle\kappa_{n}: n \in \omega\right\rangle$, then $\lambda$ is Jonsson.

Proof. Let $\mathscr{I}_{n}$ be the ideal on $\left[\kappa_{n+1}\right]^{\kappa_{n}}$. Let $G \subseteq \mathscr{P}_{f}\left([\lambda]^{\lambda}\right) / \mathscr{I}_{\omega}$ be generic and let 
$j: V \rightarrow M$ be the embedding induced by $G$. Let $F_{n}:[\lambda]^{\lambda} \rightarrow V$ be defined by $F_{n}(x)=x \cap \kappa_{n+1}$. It is easy to check that $\left[F_{n}\right]_{M}=j^{\prime \prime} \kappa_{n+1}$.

Let $\mathfrak{A}=\left(\lambda,\left(f_{i}: i \in \omega\right)\right)$ be a structure on $\lambda$ that is fully Skolemized. Without loss of generality we may assume that $\mathfrak{U} \cap \kappa_{n} \prec \mathfrak{A}$. As usual it is easy to check that $j^{\prime \prime} \kappa_{n}$ is closed under the functions $j\left(f_{i}\right)$ since $\kappa_{n}$ is closed under $f_{i}$. Thus, $\left\langle j^{\prime \prime} \kappa_{n}, j\left(f_{i}\right)\right\rangle_{i \in \omega}$ $\prec j(\mathfrak{H})$.

In $M$, let $T$ be a tree whose nodes consist of elementary substructures $\mathfrak{B}$ of $j(\mathfrak{H})$ such that, for some $n$, o.t. $\mathfrak{B}=\kappa_{n+1}$ and for all $1 \leqslant i \leqslant n+2$, o.t. $\mathscr{B} \cap \kappa_{i}=\kappa_{i-1}$. If $\mathfrak{B}$, $\mathfrak{C}$ are nodes on the tree put $\mathfrak{B}<{ }_{T} \mathbb{C}$ iff $\mathscr{B}$ is an end extension of $\mathfrak{S}$ and $|\mathfrak{B}|>|\mathfrak{C}|$.

We claim that $M \vDash T$ is not well founded.

Each $j^{\prime \prime} \kappa_{n+1}$ is a node on $T$. In $V[G]$, the sequence $\left\langle j^{\prime \prime} \kappa_{n+1}: n \in \omega\right\rangle$ is a branch through $T$. Hence, by absoluteness $T$ has a branch through it in $M$.

Let $b$ be a branch through $T$ in $M$. Then $U b<j(\mathfrak{H})$ since it is a union of elementary substructures of $j(\mathfrak{A})$. It is a proper elementary substructure since $M \vDash\left|\bigcup b \cap \kappa_{1}\right|=\kappa_{0}$.

Thus $M \vDash j(\mathfrak{U})$ has a proper elementary substructure. So $V \vDash \mathfrak{H}$ has a proper elementary substructure.

There are many natural open questions about these towers of ideals. We mention two:

Question 1. Is " $\boldsymbol{\kappa}_{\omega}$-Jonsson" equivalent to the existence of a precipitous tower of ideals on a subsequence of the $\left\langle\boldsymbol{\kappa}_{n}: n \in \omega\right\rangle$ ?

M. Magidor and the author have some partial positive results in this direction.

Question 2. If $\left\langle\mathscr{I}_{n}: n \in \omega\right\rangle$ is a tower of countably complete ideals, $\mathscr{I}_{n}$ an ideal on $\left[\boldsymbol{\kappa}_{n+1}\right]^{\boldsymbol{\aleph}_{n}}$ and the tower is $\boldsymbol{\aleph}_{2}$-saturated, is the tower precipitous?

This would be useful if it were true since it would reduce the problem of making a tower of ideals precipitous to a local problem.

There is a criterion for precipitousness that is an easy generalization of [J-P].

If $\left\langle\mathscr{I}_{n}: n \in \omega\right\rangle$ is a tower of ideals we can play the following game:

White and Black alternate playing a decreasing sequence of $\mathscr{I}_{\omega}$ positive elements of $\mathscr{P}_{f}\left([\lambda]^{\lambda}\right)$.

\begin{tabular}{lrrr} 
White & $X_{0}$ & $X_{2}$ & $\cdots$ \\
\hline Black & $X_{1}$ & $X_{3}$ & $\cdots$ \\
$X_{0} \supseteq X_{1} \supseteq X_{2} \supseteq$ & $\cdots$ &
\end{tabular}

Black wins iff there is an element $x \in[\lambda]^{\lambda}$ such that $x \in \bigcap_{n \in \omega} X_{n}$. (Black is the good guy.)

REMARK. $\mathscr{I}_{\omega}$ is precipitous iff White does not have a winning strategy in this game.

We now mention a theorem of Woodin that uses towers of ideals in its proof. (See appendix for the proof.)

Theorem (Woodin). Suppose $\mathscr{I}$ is a precipitous ideal on $\left[\boldsymbol{\kappa}_{\omega}\right]^{\boldsymbol{\aleph}_{\omega}}$ that is $\boldsymbol{\aleph}_{n}$-complete, $\boldsymbol{\aleph}_{k}$-saturated and $\boldsymbol{\aleph}_{\omega}$ is a strong limit, then $2^{\boldsymbol{N}_{0}} \geqslant \boldsymbol{\aleph}_{n}$. 
These axioms also show some nontrivial model-theoretic facts, e.g.,

PROPOSITION 30. Let $L$ be a countable similarity type, $\psi$ a formula in $L_{\kappa, \omega}$ and $A$ a fragment of $L_{\kappa, \omega},|A|<\kappa$, containing $\psi$ and skolem functions.

Suppose $\psi$ is categorical in power $\kappa$ and that there is a $\kappa$-complete, $\kappa$-centered ideal on $\left[\kappa^{+}\right]^{\kappa}$. Then any two models of $\psi$ of power $\kappa^{+}$have isomorphic A-elementary submodels of cardinality $\boldsymbol{\kappa}^{+}$.

The proof of this proposition is very similar to that of Proposition 28.

In conclusion, the author believes these ideals provide a basis for settling many classical open questions in Set Theory. The axioms need a considerable amount of work before they can be presented in an intuitive way as symmetry principles to the "working mathematician," but they are readily accessible to a literate set theorist.

Appendix. Two theorems by Magidor and Woodin, one each. (See the introduction about our general assumptions about ideals.)

THEOREM (WoOdiN). If $\boldsymbol{\aleph}_{\omega}$ is a strong limit and there is an $\boldsymbol{\aleph}_{n}$-complete, precipitous, $\boldsymbol{\aleph}_{k}$-saturated ideal $\mathscr{I}$ on $\left[\boldsymbol{\aleph}_{\omega}\right]^{\boldsymbol{\aleph}_{\omega}}$, then $2^{\boldsymbol{\aleph}_{0}} \geqslant \boldsymbol{\aleph}_{n}$.

Proof. Let $\left\langle\kappa_{n}: n \in \omega\right\rangle$ be defined in the usual way. Then, letting $\pi_{n}:\left[\aleph_{\omega}\right]^{\kappa_{\omega}} \rightarrow$ $\left[\kappa_{n+1}\right]^{\leqslant \kappa_{n+1}}$ by $\pi_{n}(x)=x \cap \kappa_{n+1}$, we can project $\mathscr{I}$ onto an ideal $\mathscr{I}_{n}$ on $\left[\kappa_{n+1}\right] \leqslant \kappa_{n+1}$. By Lemma 5, $\mathscr{I}_{n}$ concentrates on $\left[\kappa_{n+1}\right]^{\kappa_{n}}$.

We now apply standard large cardinal machinery. If $G \subseteq \mathscr{P}\left(\left[\aleph_{\omega}\right]^{\aleph_{\omega}}\right) / \mathscr{I}$ is generic, then $G$ induces an ultrafilter $G_{n}$ on $\left[\kappa_{n+1}\right]^{\kappa_{n}}$. We can form $M_{n}=V^{\left[\kappa_{n+1}\right]^{\kappa_{n}}} / G_{n}$ and $j_{n}: V \rightarrow M_{n}$. The following lemma is standard:

LEMma. There are embeddings $i_{n}, k_{n}$ so that the following diagram commutes:

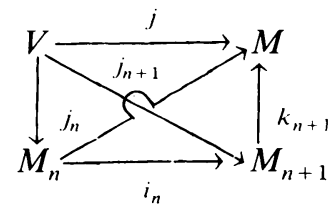

Further,

(a) $\operatorname{crit}\left(i_{n}\right)>\kappa_{n+1}$,

(b) $\operatorname{crit}\left(k_{n}\right)=\operatorname{crit}\left(i_{n}\right)$.

We let $M_{\infty}=\lim _{\rightarrow}\left\langle M_{n}: n \in \omega\right\rangle$ and $k_{\infty}: M_{\infty} \rightarrow M$ be the map induced by the $\left\langle k_{n}: n \in \omega\right\rangle$. It is clear that the following diagram commutes:

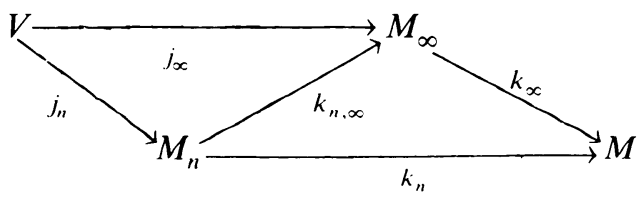

for canonical embeddings $j_{\infty}, k_{\infty}$ and $k_{n, \infty}$. 
Claim. $j^{\prime \prime} \boldsymbol{\aleph}_{\omega} \notin M_{\infty}$.

ProOF (SKeTCh). If $j^{\prime \prime} \boldsymbol{\aleph}_{\omega} \in M_{\infty}$, then $j^{\prime \prime} \boldsymbol{\aleph}_{\omega}=[F]_{M_{n}}$ where $F:\left[\kappa_{n+1}\right]^{\kappa_{n}} \rightarrow V$, for some $n$. Choose $m$ so large that

$$
\kappa_{n+1} \stackrel{\kappa^{\prime \prime}}{\stackrel{2}{2}}<\kappa_{m} .
$$

Let $F^{\prime}:\left[\kappa_{n+1}\right]^{\kappa_{n}} \rightarrow V$ be defined by $F^{\prime}(x)=F(x) \cap \kappa_{m+1}$. Let $I:\left[\kappa_{m+1}\right]^{\kappa_{m} \rightarrow V}$ be defined by $I(x)=x$. Then $[I]_{M_{x}}=j^{\prime \prime} \kappa_{m}$. Hence $\left\{y: F^{\prime}\left(y \cap \kappa_{n+1}\right)=y\right\} \in G_{m}$. But, by fineness of the ideal $\mathscr{I}_{m},\left|\left\{y: F^{\prime}\left(y \cap \kappa_{n+1}\right)=y\right\}\right| \geqslant \kappa_{m}$. Hence, $F^{\prime}$ maps $\left[\kappa_{n+1}\right]^{\kappa_{n}}$ onto a set of size $\kappa_{m}$, a contradiction.

We now use the following theorem due to Shelah [Sh]:

TheOREM (Shelah). If $\boldsymbol{\aleph}_{\omega}$ is a strong limit, then $2^{\boldsymbol{\aleph}_{\omega}}<\boldsymbol{\aleph}_{\left(2^{\left.\mathrm{N}_{0}\right)}\right.}$..

Consider the map $k_{\infty}: M_{\infty} \rightarrow M$.

Claim. $\operatorname{crit}\left(k_{\infty}\right) \geqslant \boldsymbol{\kappa}_{\kappa_{0}}$.

PROOF. Since $j\left(\boldsymbol{\aleph}_{\omega}\right)=\boldsymbol{\aleph}_{\omega}$ and $\mathscr{P}\left(\left[\boldsymbol{\aleph}_{\omega}\right]^{\boldsymbol{\aleph}_{\omega}}\right) / \mathscr{I}$ preserves cardinals above $\boldsymbol{\aleph}_{\omega}$, if $\omega \leqslant \alpha<\kappa_{0}$ then $j\left(\boldsymbol{\aleph}_{\alpha}^{V}\right)=\boldsymbol{\aleph}_{\alpha}^{V}$. Further, $k_{\infty} \circ j_{\infty}\left(\boldsymbol{\aleph}_{\alpha}\right) \geqslant \boldsymbol{\aleph}_{\alpha}$. Since $j=k_{\infty} \circ j_{\infty}$, we get $k_{\infty}\left(\boldsymbol{\aleph}_{\alpha}\right)=\boldsymbol{\aleph}_{\alpha}$ for $\omega \leqslant \alpha<\kappa_{0}$.

Suppose $\left|\mathscr{P}\left(\boldsymbol{\aleph}_{\omega}\right)\right|<\boldsymbol{\aleph}_{\kappa_{0}}$ then $M_{\infty} \vDash \mathscr{P}\left(\boldsymbol{\aleph}_{\omega}\right)<\boldsymbol{\aleph}_{\kappa_{0}}$. Then $k_{\infty}\left(\mathscr{P}\left(\boldsymbol{\aleph}_{\omega}\right)^{M_{\infty}}\right)=$ $\mathscr{P}\left(\boldsymbol{\aleph}_{\omega}\right)^{M_{x}}$. But then $\mathscr{P}\left(\boldsymbol{\aleph}_{\omega}\right)^{M_{x}}=\mathscr{P}\left(\boldsymbol{\aleph}_{\omega}\right)^{M}$, a contradiction.

Thus $\left|\mathscr{P}\left(\boldsymbol{\kappa}_{\omega}\right)\right|>\boldsymbol{\kappa}_{\kappa_{0}}$. Hence $\left(2^{\kappa_{0}}\right)^{+}>\kappa_{0}$ by Shelah's theorem, and thus $2^{\kappa_{0}} \geqslant \kappa_{0}$.

We now turn to a theorem of Magidor.

THEOREM. There is no $\boldsymbol{\aleph}_{n}$-saturated ideal $\mathscr{I}$ on $\left[\boldsymbol{\aleph}_{\omega+1}\right]^{\boldsymbol{\kappa}_{\omega+1}}$.

Proof. Suppose such an $I$ exists. We first prove the following claim:

Claim. If $\mathfrak{A}$ is a structure of cardinality $\boldsymbol{\kappa}_{\omega+1}$ with domain including $\boldsymbol{\kappa}_{\omega+1}$ then there is a proper elementary substructure $\mathfrak{B} \prec \mathfrak{A}$ such that $\mathfrak{B} \cap \boldsymbol{\aleph}_{\omega+1}$ is $\omega$-closed and $|\mathfrak{B}|=\boldsymbol{\kappa}_{\omega+1}$.

Proof of Claim. Let $j: V \rightarrow M$ be the elementary embedding induced by $\mathscr{I}$. Consider the sequence $\left\langle\kappa_{n}: n \in \omega\right\rangle$. This is a subsequence of the $\left\langle\boldsymbol{\kappa}_{i}: i \in \omega\right\rangle$, since, by earlier arguments, $j\left(\boldsymbol{\aleph}_{\omega+1}^{V}\right)=\boldsymbol{\aleph}_{\omega+1}^{V}, j\left(\boldsymbol{\aleph}_{\omega}^{V}\right)=\boldsymbol{\aleph}_{\omega}^{V}$ and $\mathscr{P}\left(\boldsymbol{\aleph}_{\omega}\right)^{V} \subseteq M$. Without loss of generality, $\mathfrak{A}$ is fully skolemized and $\mathfrak{A}$ has a reiation " $\varepsilon^{\mathfrak{l l}}$ " defined on it and with respect to $\varepsilon^{\text {th }}$ is an elementary substructure of some $H(\lambda)$ for a very large $\lambda$. As we argued in Proposition $16, j^{\prime \prime} \mathfrak{A}$ is an elementary substructure of $j(\mathfrak{H})$ such that, for all $m \in \omega$, o.t. $\left(j^{\prime \prime} \mathfrak{A} \cap \aleph_{m}^{M}\right)=\aleph_{m}^{V}$. By the $\aleph_{n}$-saturation of $\mathscr{I}$ we get that, for large enough $m, \aleph_{m}^{V}$ is a cardinal in $M$.

Thus in $M, j(\mathfrak{A})$ has an elementary substructure $\mathfrak{B}$ such that $\mid(j(\mathfrak{H})-\mathfrak{B}) \cap$ $\boldsymbol{\aleph}_{\omega+1} \mid \geqslant \boldsymbol{\aleph}_{\omega}$ and for some $n_{0}>1$ for all large enough $m>n_{0}$ o.t. $\mathscr{B} \cap \boldsymbol{\aleph}_{m}$ is a cardinal. Let $\mathfrak{B}^{*}$ be the elementary substructure of $\mathfrak{A}$ generated by $\mathfrak{B} \cup \boldsymbol{\aleph}_{n_{0}}$.

We will show that, for each $m>n_{0}, \sup \mathfrak{B}^{*} \cap \boldsymbol{\aleph}_{m}=\sup \mathfrak{B} \cap \boldsymbol{\aleph}_{m}$. Let $\alpha \in \mathfrak{H}^{*} \cap$ $\aleph_{m}$. Then $\alpha=f(\vec{\beta}, \vec{\gamma})$ for some $\vec{\beta} \in \mathfrak{B}, \vec{\gamma} \in\left(\boldsymbol{\aleph}_{n}\right)^{<\omega}$ and some $f$ in $\mathscr{U}$. Let $\delta=\sup _{\vec{\gamma} \in\left(\boldsymbol{\aleph}_{n}\right)^{<\omega}} f(\vec{\beta}, \vec{\gamma}) \cap \boldsymbol{\aleph}_{m}$. Then $\delta \in \mathfrak{B}$ and $\delta>\alpha$. Hence $\mathfrak{B}^{*}$ is a proper elementary substructure of $\mathfrak{A}$. 
We now show that $\mathfrak{B}^{*} \cap \boldsymbol{\aleph}_{\omega+1}$ is countable closed. Let $\alpha<\boldsymbol{\aleph}_{\omega+1}$ be the least limit point of $\mathfrak{B}^{*}$ of cofinality $\omega$ such that $\alpha \notin \mathfrak{B}^{*}$. Let $\alpha^{*}$ be the least element of $\mathfrak{B}^{*}$ above $\alpha$. If $\operatorname{cof}\left(\alpha^{*}\right)=\omega$ in $\mathfrak{B}^{*}$, then in $\mathfrak{B}^{*}$ we can find a sequence $\left\langle\alpha_{n}\right.$ : $n \in \omega\rangle \in \mathfrak{B}^{*}$ cofinal in $\alpha^{*}$. But, since $\omega \subseteq \mathfrak{B}^{*}$, each $\alpha_{n} \in \mathfrak{B}$, so $\mathfrak{B}^{*} \cap \boldsymbol{\aleph}_{\omega+1}$ is cofinal in $\alpha^{*}$; this contradicts our choice of $\alpha^{*}$.

Suppose that $\mathfrak{B}^{*} \vDash \operatorname{cof}\left(\alpha^{*}\right)=\boldsymbol{\aleph}_{m}$. Let $\left\langle\alpha_{\gamma}: \gamma\left\langle\boldsymbol{\aleph}_{m}\right\rangle \in \mathfrak{B}^{*}\right.$ be such that $\mathfrak{B}^{*} \vDash$ $\left\langle\alpha_{\gamma}: \gamma\left\langle\boldsymbol{N}_{m}\right\rangle\right.$ is cofinal in $\alpha^{*}$. Then $\left\langle\alpha_{\gamma}: \gamma\left\langle\mathfrak{B}^{*} \cap \boldsymbol{N}_{m}\right\rangle\right.$ is cofinal in sup $\mathfrak{B}^{*} \cap \boldsymbol{\alpha}^{*}$ $=\alpha$. Hence $\operatorname{cof}(\alpha)=\operatorname{cof}\left(\sup \mathfrak{B}^{*} \cap \boldsymbol{\aleph}_{n}\right)$. But $\mathfrak{B}^{*} \cap \boldsymbol{\aleph}_{n}$ has order type a regular cardinal $>\omega$, a contradiction! This proves the claim.

To see that the claim implies the theorem we divide the points of cofinality $\omega$ in $\boldsymbol{\aleph}_{\omega+1}$ into $\boldsymbol{\aleph}_{\omega+1}$ disjoint stationary sets $\left\langle\dot{A}_{\alpha}: \alpha<\boldsymbol{\aleph}_{\omega+1}\right\rangle$. Let $\mathfrak{A} \prec H(\lambda)$ where $|\mathfrak{A}|=\boldsymbol{\aleph}_{\omega+1}$ and $\left\langle A_{\alpha}: \alpha<\boldsymbol{\aleph}_{\omega+1}\right\rangle \cup \boldsymbol{\aleph}_{\omega+1} \subseteq \mathfrak{A}$. Let $\mathfrak{B} \prec \mathfrak{A}$ be an $\omega$-closed elementary substructure of $\mathfrak{A}$ such that $\left\langle A_{\alpha}: \alpha<\aleph_{\omega+1}\right\rangle \in \mathfrak{B}$ and $\aleph_{\omega+1}-\mathfrak{B} \neq \varnothing$. Then, by elementarity, $\mathfrak{B} \cap\left\{\beta<\boldsymbol{\aleph}_{\omega+1}\right.$ : $\left.\operatorname{cof}(\beta)=\omega\right\}=\bigcup_{\alpha \in \mathfrak{B} \cap \boldsymbol{\aleph}_{\omega+1}} A_{\alpha}$. Hence $\bigcup_{\alpha \in \Psi \cap \aleph_{\omega+1}} A_{\alpha}$ includes an $\omega$-closed unbounded set. Let $\delta \in \aleph_{\omega+1}-\mathfrak{B}$. Then $A_{\delta} \cap \bigcup_{\alpha \in \mathbb{H} \cap \aleph_{\omega+1}} A_{\alpha}=\varnothing$, but $A_{\delta}$ was supposed to be stationary. This contradiction proves the theorem.

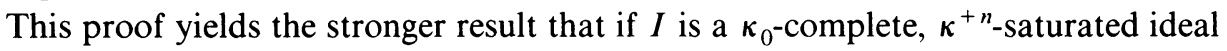
on $[\lambda]^{\gamma}$ for some $\gamma$ then $\lambda \leqslant \kappa^{+\omega}$.

To see this we note that by the saturation condition the $\left\langle\kappa_{n}: n \in \omega\right\rangle$ are a subsequence of the $\left\langle\kappa^{+n}: n \in \omega\right\rangle$. Hence, $\sup \left\langle\kappa_{i}: i \in \omega\right\rangle=\kappa^{+\omega}$ and $\kappa^{+\omega}$ is a fixed point of the generic embedding.

Now we argue as above to prove the theorem.

\section{BIBLIOGRAPHY}

[B] J. E. Baumgartner, A generic graph construction, J. Symbolic Logic (to appear).

[B-T] J. E. Baumgartner and A. D. Taylor, Suturation of ideals in generic extensions. II, Trans. Amer. Math. Soc. 271 (1982), 587-609.

[F1] M. Foreman, More saturated ideals, Cabal Seminar 1979-1981, Lecture Notes in Math., vol. 1019, Springer-Verlag, 1983.

[F2] _ Large cardinals and strong model-theoretic transfer properties, Trans. Amer. Math. Soc. 272 (1982), 427-463.

[F-L] M. Foreman and R. Laver, A graph transfer property (to appear).

[F-M1] M. Foreman and M. Magidor, $\square_{k}$ is consistent with a $k^{+}$-saturated ideal on $K$ (to appear).

[F-M2] _ An $\boldsymbol{\aleph}_{2}$-saturated ideal on $\boldsymbol{\aleph}_{\omega_{1}}+1$.

[G] H. Gaifman, Elementary embeddings of models of set-theory and certain subtheories, Axiomatic Set Theory, Proc. Sympos. Pure Math., Vol. 13, Part II (T. Jech, ed.), Amer. Math. Soc., Providence, R.I., 1971, pp. 33-101.

[Ga] F. Galvin, Chromatic numbers of subgraphs, Period. Mat. Hungar. 4 (1973), 117-119.

[J1] T. Jech, Set theory, Academic Press, New York, 1978.

[JMMP] T. Jech, M. Magidor, W. Mitchell and K. Prikry, Precipitous ideals, J. Symbolic Logic 45 (1980), 1-8.

[J-P] T. Jech and K. Prikry, On ideals of sets and the power set operation, Bull. Amer. Math. Soc. 82 (1976), 593-595.

[K1] K. Kunen, Some applications of iterated ultrapowers in set theory, Ann. of Math. Logic 1 (1970), 179-227.

[K2] Saturated ideals, J. Symbolic Logic 43 (1978), 65-76.

[L] R. Laver, Saturated ideals and non-regular ultrafilters. Proc. Bernays Conference (Patras, Greece, 1980), North-Holland, 1982.

[M1] M. Magidor, Precipitous ideals and $\Sigma_{4}^{1}$-sets. 
[Ma] A. R. D. Mathias, Happy families, Ann. of Math. Logic 12 (1977), 59-111.

[M-S] J. Mycielski and S. Swierczkowski, On Lebesgue measureability and the axiom of determinateness. Fund. Math. 54 (1964).

[S] J. Silver, The consistency of the GCH with the existence of a measurable cardinal, Axiomatic Set Theory, Proc. Sympos. Pure Math., Vol. 13, Part I. Amer. Math. Soc., Providence, R.I., 1971, pp. 391-395.

[Sh] S. Shelah, unpublished.

[Sh1] __ Proper forcing, Lecture Notes in Math., vol. 940, Springer-Verlag, 1982.

[Sh2] __ Infinite Abelian groups Whitehead problem and some constructions, Israel J. Math. 13 (1977).

[So1] R. M. Solovay, A model of set theory in which every set of reals is Lehesgue measurable, Ann. of Math (2) 92 (1970), 1-56.

[So2] _. Real-valued measurable cardinals, Axiomatic Set Theory, Proc. Sympos. Pure Math.. Vol. 13, Part I, Amer. Math. Soc., Providence, R. I., 1971, pp. 397-428.

[SRK] R. M. Solovay, N. N. Reinhardt and A. Kanamori, Strong axioms of infinity and elementary embeddings, Ann. of Math. Logic 13 (1978), 73-116.

[W1] W. H. Woodin, Discontinuous homomorphisms of $C(X)$ and set theory (to appear).

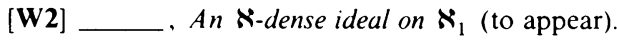

Department of Mathematics, Hebrew University of Jerusalem, Jerusalem, Israel

Department of Mathematics, Rutgers University, New Brunswick, New Jersey 08903

Current address: Department of Mathematics, California Institute of Technology, Pasadena, California 91125 